\title{
On the physics of frequency domain controlled source electromagnetics in shallow water, 2: transverse anisotropy
}

\author{
Alan D. Chave, ${ }^{1}$ Johan Mattsson ${ }^{2}$ and Mark E. Everett ${ }^{3}$ \\ ${ }^{1}$ Department of Applied Ocean Physics and Engineering, Woods Hole Oceanographic Institution, Woods Hole, MA 02543, USA. E-mail: achave@whoi.edu \\ ${ }^{2}$ PGS, S-164-46 Kista, Stockholm, Sweden \\ ${ }^{3}$ Department of Geology and Geophysics, Texas A\&M University, College Station, TX 77845, USA
}

Accepted 2017 August 24. Received 2017 August 21; in original form 2017 May 23

\begin{abstract}
SUMMAR Y
In recent years, marine controlled source electromagnetics (CSEM) has found increasing use in hydrocarbon exploration due to its ability to detect thin resistive zones beneath the seafloor. It is the purpose of this paper to evaluate the physics of CSEM for an ocean whose electrical thickness is comparable to or much thinner than that of the overburden using the in-line configuration through examination of the elliptically-polarized seafloor electric field, the time-averaged energy flow depicted by the real part of the complex Poynting vector, energy dissipation through Joule heating and the Fréchet derivatives of the seafloor field with respect to the sub-seafloor conductivity that is assumed to be transversely anisotropic, with a verticalto-horizontal resistivity ratio of $3: 1$. For an ocean whose electrical thickness is comparable to that of the overburden, the seafloor electromagnetic response for a model containing a resistive reservoir layer has a greater amplitude and reduced phase as a function of offset compared to that for a halfspace, or a stronger and faster response, and displays little to no evidence for the air interaction. For an ocean whose electrical thickness is much smaller than that of the overburden, the electric field displays a greater amplitude and reduced phase at small offsets, shifting to a stronger amplitude and increased phase at intermediate offsets, and a weaker amplitude and enhanced phase at long offsets, or a stronger and faster response that first changes to stronger and slower, and then transitions to weaker and slower. By comparison to the isotropic case with the same horizontal conductivity, transverse anisotropy stretches the Poynting vector and the electric field response from a thin resistive layer to much longer offsets. These phenomena can be understood by visualizing the energy flow throughout the structure caused by the competing influences of the dipole source and guided energy flow in the reservoir layer, and the air interaction caused by coupling of the entire sub-seafloor resistivity structure with the sea surface. The Fréchet derivatives are dominated by preferential sensitivity to the vertical conductivity in the reservoir layer and overburden at short offsets. The horizontal conductivity Fréchet derivatives are weaker than to comparable to the vertical derivatives at long offsets in the substrate. This means that the sensitivity to the horizontal conductivity is present in the shallow parts of the subsurface. In the presence of transverse anisotropy, it is necessary to go to higher frequencies to sense the horizontal conductivity in the overburden as compared to an isotropic model with the same horizontal conductivity. These observations in part explain the success of shallow towed CSEM using only measurements of the in-line component of the electric field.
\end{abstract}

Key words: Electrical properties; Electrical anisotropy; Marine electromagnetics.

\section{INTRODUCTION}

Pioneering measurements by Cox et al. (1978) showed that the natural background electric field at frequencies in the vicinity of $1 \mathrm{~Hz}$ in the deep ocean is extremely low $\left(\sim 1 \mathrm{pV} \mathrm{m}^{-1}\right)$, suggesting that the weak fields induced within Earth by a near-seafloor artificial source could be detected at large (many $\mathrm{km}$ ) source-receiver offsets. This led to the development of a new geophysical exploration method based on seabed-to-seabed propagation of low-frequency electromagnetic (EM) fields from a horizontal electric dipole (HED) that is preferentially sensitive to resistive rather than conductive material. 
It has been well known since the 1930s that petroleum-bearing layers in clastic sedimentary formations are thin and commonly but not ubiquitously resistive compared to the substrate in which they are embedded. In 2000, it was demonstrated that a producing offshore petroleum field could be mapped with the same marine controlled source electromagnetic (CSEM) technique and apparatus utilized in academia over the preceding two decades (Eidesmo et al. 2002; Ellingsrud et al. 2002; Bhuiyan 2009), leading to commercialization of the technology. Constable \& Srnka (2007) and Constable (2010) provide historical reviews, whereas MacGregor $\&$ Tomlinson (2014) give a recent technical overview. In addition to conventional seafloor receivers, a towed streamer system has been developed and commercialized for hydrocarbon exploration in shallow water. A description of this system and its performance can be found in Mattsson et al. (2013), Zhdanov et al. (2014), and McKay et al. (2015).

It must be recognized that the quantitative interpretation of marine CSEM data over petroleum-bearing formations will typically require 2-D surveys and 2-D or 3-D modeling that accommodates transverse anisotropy and bathymetry. However, considerable insight into the physics of marine CSEM can be obtained from 1-D models. In a recent paper, Chave et al. (2017; hereafter Paper I) utilized a 1-D isotropic approach to provide insight into the diffusive physics of marine CSEM when the so-called airwave [more appropriately termed the air interaction; Andréis \& MacGregor (2008)] is important through analysis of the Poynting vector, Joule heating, elliptically-polarized representation of the seafloor electric field and Fréchet derivatives of the seafloor electric field with respect to the subsurface conductivity. Paper I showed that the deep water (ocean layer electrically much thicker than the overburden) seafloor EM response for a model containing a resistive reservoir layer has a greater amplitude and reduced phase as a function of offset compared to that for a halfspace, or a stronger and faster response. For an ocean whose electrical thickness is comparable to or much smaller than that of the overburden, the electric field displays a greater amplitude and reduced phase at small offsets, shifting to a stronger amplitude and increased phase at intermediate offsets, and a weaker amplitude and enhanced phase at long offsets, or a stronger and faster response that first changes to stronger and slower, and then transitions to weaker and slower. These transitions were explained through the energy flow in the structure caused by the competing influences of the dipole source and guided energy flow in the reservoir layer, and the air interaction caused by inductive coupling of the entire sub-seafloor resistivity structure with the sea surface. A stronger and faster response occurs when guided energy flow is dominant, whereas a weaker and slower response occurs when the air interaction is dominant. However, at intermediate offsets for some models, the air interaction can partially or fully reverse the direction of energy flux in the substrate and reservoir layer toward rather than away from the source, resulting in a stronger and slower response. The Fréchet derivatives were shown to be dominated by preferential sensitivity to the reservoir layer conductivity for all water depths, but also display a shift with offset from the galvanic to the inductive mode in the underburden and overburden due to the interplay of guided energy flow and the air interaction. These observations suggest that shallow water CSEM surveys based only on the in-line orientation of source and receivers can resolve both the horizontal and vertical conductivities, in contrast to its behavior in deep water.

This paper extends Paper I by including transverse anisotropy in the overburden and underburden. The reservoir layer is taken to be isotropic, as it is very difficult to resolve anisotropy within it even

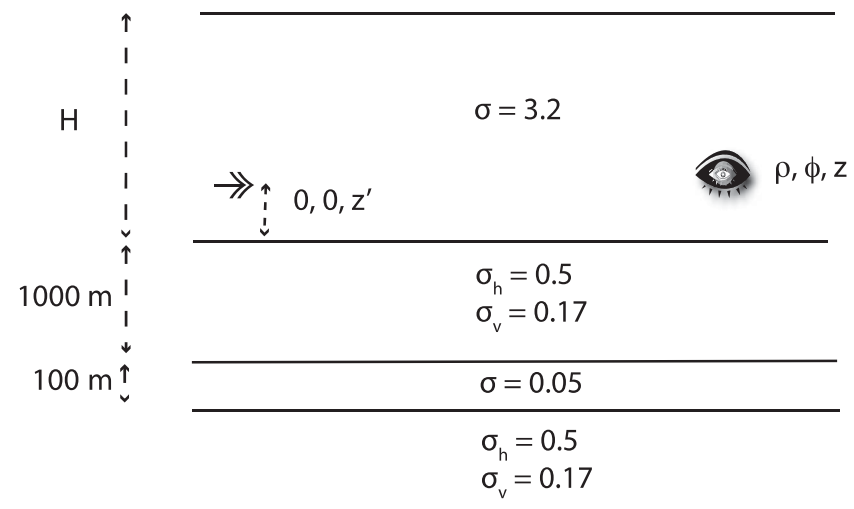

Figure 1. The canonical reservoir model used throughout this paper. The source (double arrow) is located at $\left(0,0, z^{\prime}\right)$ and the observation point (eye) is at $(\rho, 0, z)$, where the middle coordinate is the azimuth $\varphi$. The ocean layer of thickness $H$ and conductivity $3.2 \mathrm{~S} \mathrm{~m}^{-1}$ is overlain by an insulating atmosphere and underlain by a two layer structure in turn underlain by a half-space. The overburden and underburden have a horizontal conductivity of $0.5 \mathrm{~S} \mathrm{~m}^{-1}$ (resistivity of $2 \Omega-\mathrm{m}$ ), a vertical conductivity of $0.17 \mathrm{~S} \mathrm{~m}^{-1}$ (resistivity of $6 \Omega-\mathrm{m}$ ) and the reservoir layer between 1000 and $1100 \mathrm{~m}$ depth has an isotropic conductivity of $0.05 \mathrm{~S} \mathrm{~m}^{-1}$ (resistivity of $20 \Omega-\mathrm{m}$ ).

in the presence of laminated internal structures (Brown et al. 2012). The importance of transverse anisotropy in modeling and inversion of marine CSEM data is well established (e.g. Newman et al. 2010; Ramananjaona et al.2011; MacGregor \& Tomlinson 2014). Further, the presence of transverse anisotropy in clastic sedimentary formations is pervasive on the length scales sensed by marine CSEM, and is commonly explained by layering of electrically-distinct lithologies, although intrinsic fabric anisotropy is sometimes observed in shale formations. The vertical resistivity is typically higher than the horizontal resistivity by a factor of 2-4, and values of over 10 have been reported (Anderson et al. 1994; Bouchara et al. 2015).

The remainder of this paper is organized into four sections. Section 2 summarizes the diffusive physics of marine CSEM in transversely anisotropic media, Poynting's Theorem for a time harmonic field, the elliptical representation of the electric field and the Frèchet derivatives of the EM field with respect to conductivity as a function of depth, drawing on Paper I for most of the details. Section 3 presents model results based on a canonical transversely anisotropic 1-D reservoir model for both the seafloor and shallow towed configurations. Section 4 contains a discussion of the results. Section 5 gives the conclusions.

\section{THEORY}

The canonical reservoir model used in this paper is shown in Fig. 1. The model comprises a 100-m-thick reservoir layer centered at $1050 \mathrm{~m}$ depth in a substrate with a horizontal electrical conductivity of $0.5 \mathrm{~S} \mathrm{~m}^{-1}$ (resistivity of $2 \Omega-\mathrm{m}$ ) and a vertical conductivity of $0.17 \mathrm{~S} \mathrm{~m}^{-1}$ (resistivity of $6 \Omega-\mathrm{m}$ ), for a vertical to horizontal resistivity contrast of $3: 1$. The reservoir layer has an isotropic conductivity of $0.05 \mathrm{~S} \mathrm{~m}^{-1}$ (resistivity of $20 \Omega-\mathrm{m}$ ). A water layer of isotropic conductivity $3.2 \mathrm{~S} \mathrm{~m}^{-1}$ (resistivity of $0.31 \Omega-\mathrm{m}$ ) and a variable thickness $H$ overlies the substrate, and is in turn overlain by an insulating air halfspace. A point horizontal electric dipole (HED) source is located at $\left(0,0, z^{\prime}\right)$, where $z^{\prime}$ is set to $30 \mathrm{~m}$ above the seafloor for the seafloor configuration and $10 \mathrm{~m}$ below the sea surface for the shallow towed configuration, and oriented along the $x$-axis, whereas a receiver is located at $(\rho, \varphi, z)$. For the purposes of this paper, only the in-line ( $x$-directed) response is 

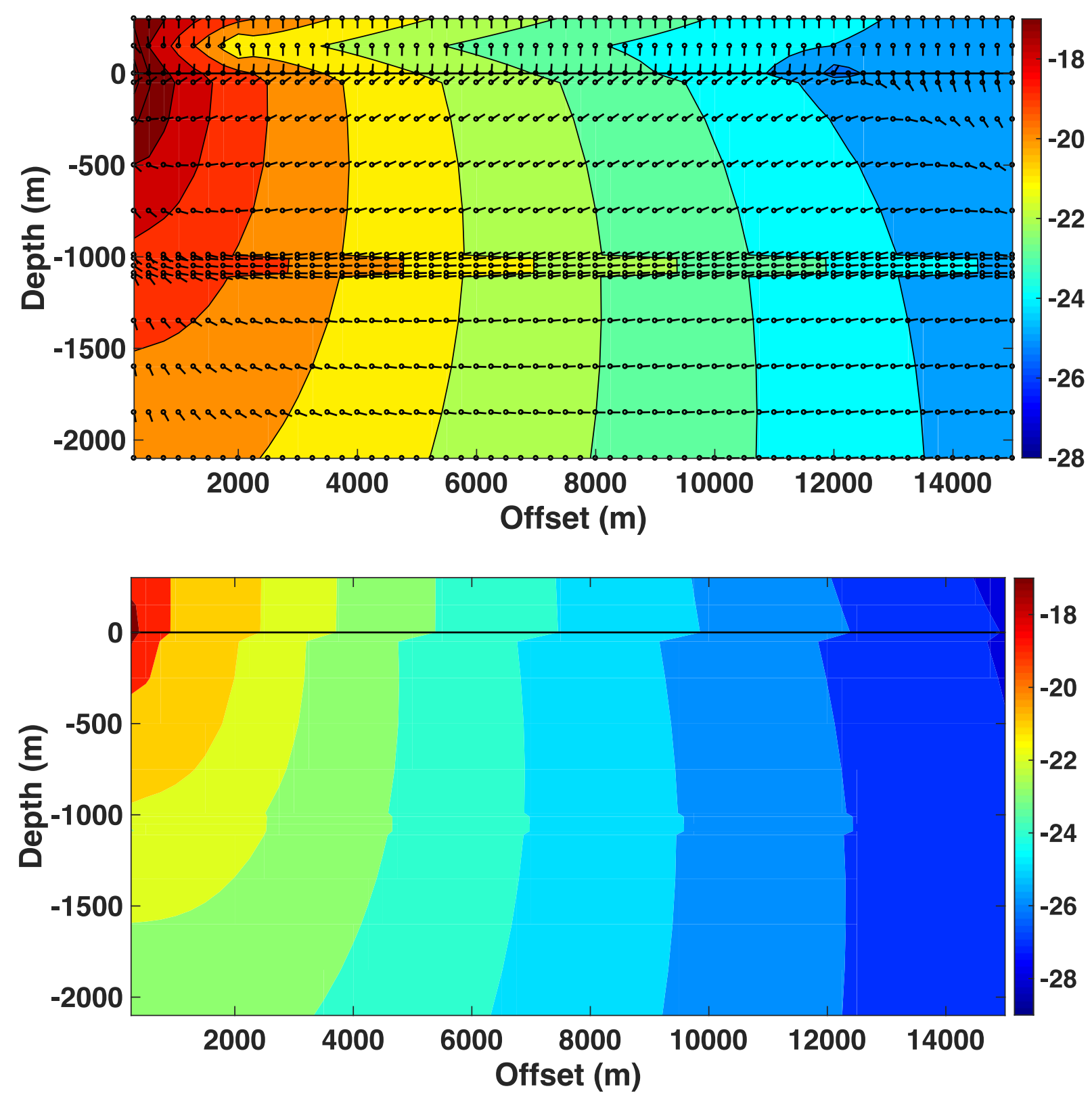

Figure 2. Contours of the logarithm (base 10) of the magnitude of the Poynting vector (top) and Joule heating (bottom) as a function of source-receiver offset and depth for the model of Fig. 1. The water depth is $300 \mathrm{~m}$, the source frequency is $0.1 \mathrm{~Hz}$ and the point source lies $30 \mathrm{~m}$ above the seafloor at the origin. The seafloor is depicted by a solid horizontal black line. The Poynting vector plot also shows the direction of energy flow at each of the small circles throughout the structure. The arrow orientations have been adjusted for the different horizontal and vertical scales.

considered, and so the receiver azimuth $\varphi$ is set to zero. The $z$-axis is positive upwards, with $z=0$ corresponding to the sea surface. Water depths of 2000, 300, and $50 \mathrm{~m}$ will be considered.

As elaborated in Paper I, the pre-Maxwell equations without displacement current are sufficient for marine CSEM, and the physics is that of diffusion driven by a continuous, temporally-periodic source. The consequence is an EM field that evolves unidirectionally forward in time at all points away from a source. Further, diffusive systems like CSEM respond to a driving source simultaneously at all locations within it, and so the EM response at a given point in a conductive medium is influenced by the resistivity of the entire structure.

Chave (2009) derived the EM fields produced by an HED source in terms of poloidal and toroidal magnetic (PM and TM) modes. The governing differential equations are given by eqs (12)-(13) in that paper; these must be modified in the presence of transverse anisotropy. The PM mode equation is unchanged, and depends only on the horizontal conductivity $\sigma_{\mathrm{h}}$. The TM mode equation becomes:

$\nabla_{\mathrm{h}}^{2} \Pi+\sigma_{\mathrm{v}} \partial_{z}\left(\partial_{z} \Pi / \sigma_{\mathrm{h}}\right)-\mu \sigma_{\mathrm{v}} \partial_{t} \Pi=\mu \Xi-\mu \sigma_{\mathrm{v}} \partial_{z}\left(\mathrm{~T} / \sigma_{\mathrm{h}}\right)$

and also depends on the vertical conductivity $\sigma_{\mathrm{v}}$. The boundary conditions are unchanged, and depend only on $\sigma_{\mathrm{h}}$. Consequently, the PM mode Green's function derived in Chave (2009) is unchanged, depending on $\sigma_{\mathrm{h}}$, whereas the TM mode Green's function is altered by replacing the term $\beta$ in the exponential terms and diffusion interaction coefficients with:

$\beta=\sqrt{\sigma_{\mathrm{h}} k^{2} / \sigma_{\mathrm{v}}-i \omega \mu \sigma_{\mathrm{h}}}$ 

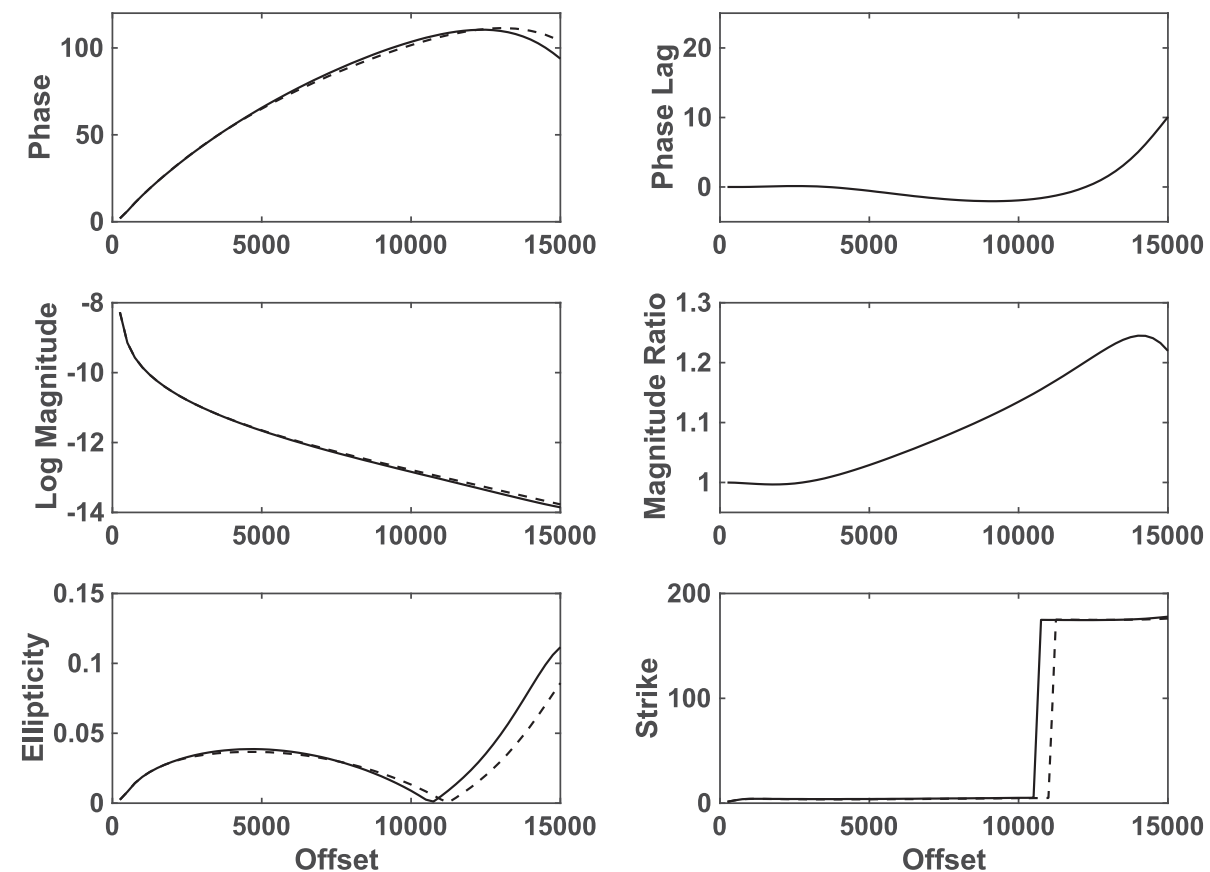

Figure 3. The polarization ellipse representation of the seafloor electric field as a function of offset from an HED point source placed at the origin but $30 \mathrm{~m}$ above the seafloor. The water depth is $300 \mathrm{~m}$ and the source frequency is $0.1 \mathrm{~Hz}$. From the upper left and proceeding counter-clockwise, the panels show the phase of the reservoir and halfspace responses, the base 10 logarithm of the magnitude of the reservoir and halfspace responses, the ellipticity, the strike of the semi-major axis in the ellipse plane, magnitude ratio of the reservoir to the halfspace response and the phase lag between the reservoir and halfspace responses. For the first four parameters, the dashed line is the reservoir result while the solid line is the halfspace result.

The EM fields within the layered structure may easily be derived by matching solutions to the homogeneous forms of the modal equations at the seafloor to the Green's functions using the boundary conditions, and may be propagated down to the depth of interest. Such solutions will be used to estimate the Poynting vector and Joule heating throughout the conductive structure.

In the absence of insight from ray concepts that pertain in wave physics, it is useful to examine the flow of energy to visualize CSEM field behavior. Chave (2009) showed from first principles that Poynting's Theorem for the pre-Maxwell equations is given by

$\nabla \cdot \mathbf{S}+\partial_{t} w_{B}+\mathbf{J} \cdot \mathbf{E}=0$,

where $\mathbf{S}=(\mathbf{E} \times \mathbf{B}) / \mu_{\mathrm{o}}$ is the Poynting vector, $w_{\mathrm{B}}=|\mathbf{B}|^{2} /\left(2 \mu_{\mathrm{o}}\right)$ is the stored energy density in the magnetic field and $\mathbf{J} \cdot \mathbf{E}$ is the energy dissipation rate density. There is no stored energy in the electric field under the pre-Maxwell approximation because electric energy is accumulated by bound charge that plays no role in EM induction. A physical interpretation follows by applying the divergence theorem to (1). In a volume of material that does not include a source, $\mathbf{S}$ represents the energy per unit area per unit time flowing into a volume of material that is balanced by the thermodynamicallyirreversible Joule heating rate and the time rate of change of energy stored in the magnetic field within the volume.

For continuous, time harmonic forcing, Chave (2009) also showed that the time average (over a complete cycle of the source) of $\boldsymbol{S}$ is equal to the real part of the complex Poynting vector $\boldsymbol{S}=\left(\mathbf{E} \times \mathbf{B}^{*}\right) /\left(2 \mu_{\mathrm{o}}\right)($ where the superscript $*$ denotes the complex conjugate; note that $\boldsymbol{S} \neq \mathbf{S}$ ) that represents the time-averaged energy flux into a volume of material that is balanced by Joule heating within it; Stratton (1941, Section 2.20) gives a similar derivation.

The EM fields produced in CSEM have a 3-D vector rather than a scalar form, and hence the standard practice of evaluating the amplitude and/or phase of a single Cartesian field component against offset or frequency is not sufficient for understanding their behavior. It is well known that a time harmonic vector field can be depicted as a polarization ellipse oriented appropriately in space, and this representation serves as a complete description of the field. Further, when an ellipse is required to depict the EM field, its Cartesian components are not independent. Chave (2009) derived a representation for elliptically-polarized EM fields that will be utilized here, and is depicted for the in-line configuration by Fig. 2 of Paper I.

Chave (1984) proved that the PM and TM mode EM fields are Fréchet differentiable, meaning that a continuous perturbation relation exists between a given EM datum and the conductivity at a given depth, with a remainder term that is second order in the model change. The Fréchet derivative relationship is

$\delta E_{x}=\int_{-\infty}^{-H} f(s) \delta \sigma(s) d s$

so that the Fréchet derivative $f(z)$ has units of volts per Siemen-m. The Fréchet derivatives were computed using a centered two point finite difference stencil operating on a 1-D forward solution whose conductivity is perturbed up or down by 5 per cent using 20 m layers from the seafloor to below the reservoir layer, and will be used to further elucidate the behavior of CSEM fields in the presence of the air interaction.

\section{MODEL RESULTS}

Fig. 2 shows the real part of the complex Poynting vector (hereafter simply Poynting vector) and Joule heating as a function of offset and depth for a source frequency of $0.1 \mathrm{~Hz}$ and a water layer thickness of $300 \mathrm{~m}$ for the seafloor configuration. The source is a point dipole with unit moment throughout this paper. At this frequency, the skin depth (the distance over which the EM field attenuates by $1 / e$ in a uniform conductor) in seawater (overburden) is $890 \mathrm{~m}$ 
(a)
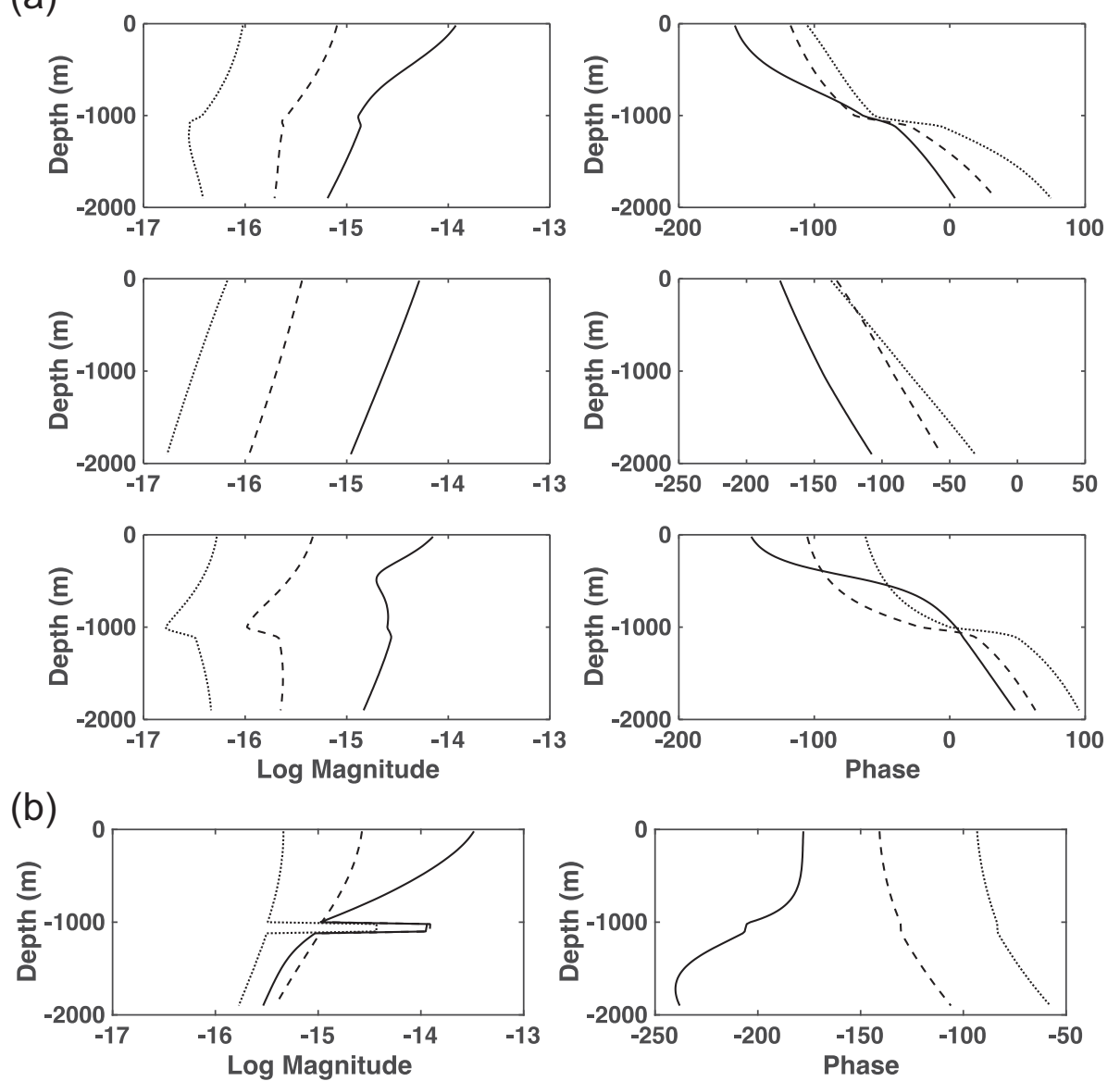

Figure 4. The Fréchet derivatives with respect to the horizontal conductivity (a) and vertical conductivity (b) for the in-line seafloor electric field as a function of depth below the seafloor for a point HED source located at the origin but $30 \mathrm{~m}$ above the seafloor. The water depth is $300 \mathrm{~m}$ and the source frequency is $0.1 \mathrm{~Hz}$. The left panels show the logarithm of the magnitude and the right panels show the phase. From top to bottom, the rows in (a) show the total Fréchet derivative, the PM mode component and the TM mode component. The different curves correspond to offsets of $3 \mathrm{~km}$ (black), $7 \mathrm{~km}$ (dashed) and $11 \mathrm{~km}$ (dotted).

(3.8 $\mathrm{km}$ for the vertical resistivity), so the receivers are about 0.34 skin depth below the sea surface and about 0.26 skin depth above the reservoir, and guided energy flow in the reservoir layer will be important to dominant. The Poynting vector and Joule heating plots show the base 10 logarithm of the magnitude of each quantity in color scale; note that the units of the Poynting vector are watts $\mathrm{m}^{-2}$ whereas the dissipation term is in watts $\mathrm{m}^{-3}$, and so the color scales are not equivalent. The color scales in Fig. 2 are identical to those for Fig. 6 of Paper I for isotropic resistivity at the same water depth and frequency. The Poynting vector plot also shows the direction of energy flow at each of the small circles throughout the structure. Fig. 2 is dominated by nearly horizontal energy flow away from the source within the reservoir layer that leaks into the overburden, decreasing monotonically in magnitude with offset. For a given offset, the Poynting vector magnitude is largest within the reservoir layer, and the tongues of energy flux extend further outward as range increases up to $\sim 7 \mathrm{~km}$ offset, and then remain of constant length. The energy flux within the water layer is primarily downward away from the seafloor, and the air interaction is apparent at the longest offsets, where the flux direction at the seafloor flips from upward to downward at $\sim 13 \mathrm{~km}$ offset. By comparison to Fig. 6 of Paper I, the Poynting vector magnitude is higher at a given offset in the presence of transverse anisotropy, and the seafloor energy flux shifts from outward to downward flux at $\sim 6.5 \mathrm{~km}$ for an isotropic model, whereas the transition occurs beyond $\sim 10 \mathrm{~km}$ in Fig. 2. A transition to downward flux in the reservoir layer is not seen at $15 \mathrm{~km}$ offset. The differences between the transversely anisotropic and isotropic models are due to the competing influence of guided energy flow in the reservoir layer and the air interaction. The air interaction is nearly identical for the two models, as it depends only on the horizontal conductivity, whereas guided energy flow depends on the vertical conductivity, and the higher resistivity in the transversely anisotropic overburden results in a stronger dipole signal at the reservoir layer. Further, the increased dissipation within the reservoir layer in Fig. 2 is more modest than in Fig. 6 of Paper I. Overall, the $300 \mathrm{~m}$ water depth transverse anisotropic response more closely resembles the deep water $(2000 \mathrm{~m})$ isotropic response at the same frequency in Fig. 3 of Paper I than the $300 \mathrm{~m}$ case.

The seafloor electric field (Fig. 3) is depicted as the generalized phase lag of the reservoir model relative to a transversely anisotropic halfspace one, and the ratio of the electric field amplitude for the reservoir model of Fig. 1 to that of a transversely anisotropic halfspace (upper and middle right panels), in both cases against source-receiver offset. The remaining panels show the ellipse amplitude/phase and orientation parameters for both the reservoir and halfspace models as described in Paper I. As a function of offset, the seafloor electric field shows a positive amplitude ratio 

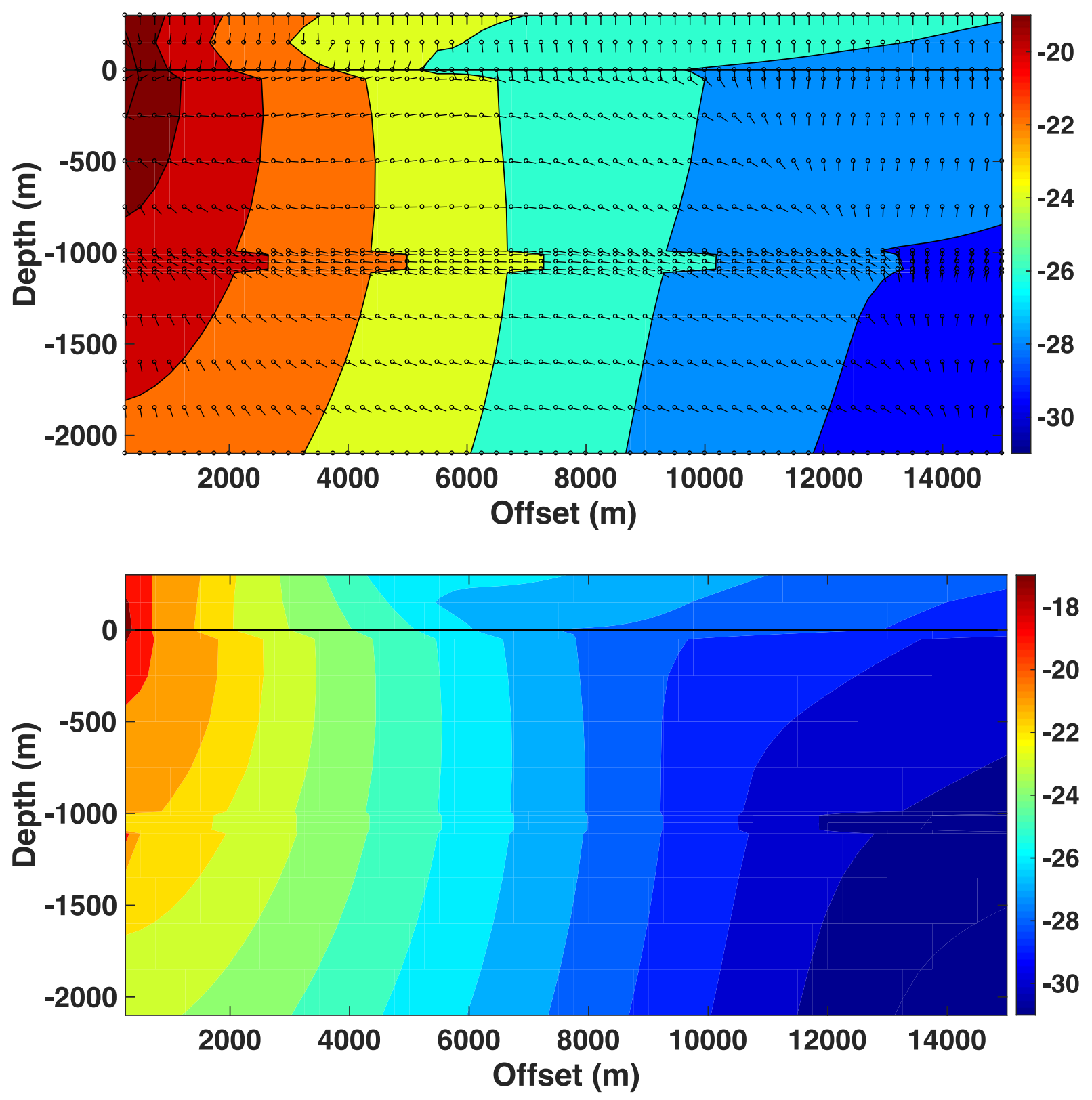

Figure 5. Contours of the logarithms (base 10) of the magnitudes of the Poynting vector (top) and Joule heating (bottom) as a function of source-receiver offset and depth for the model of Fig. 1. The water depth is $300 \mathrm{~m}$, the source frequency is $1 \mathrm{~Hz}$ and the point source lies $30 \mathrm{~m}$ above the seafloor at the origin. The seafloor is depicted by a solid horizontal black line. The Poynting vector plot also shows the direction of energy flow at each of the small circles throughout the structure. The arrow orientations have been adjusted for the different horizontal and vertical scales.

beyond $\sim 2.5 \mathrm{~km}$ and a weak phase lead over $\sim 2.5-12 \mathrm{~km}$, followed by a phase that lags the halfspace value out to the longest offset. An amplitude ratio greater than unity and a negative phase lag indicate that the response of the reservoir model is stronger and faster compared to that of a halfspace. Consequently, the reservoir response is initially stronger and slightly faster than the halfspace response, then becomes stronger and slower beyond $\sim 12 \mathrm{~km}$ offset. This behavior is accompanied by concomitant changes in the ellipse shape. By comparison with Fig. 7 in Paper I, all of the polarization ellipse parameters in Fig. 6 resemble their isotropic counterparts stretched to larger offsets.

Fig. 4(a) shows the in-line electric field Fréchet derivatives with respect to the horizontal conductivity, and their PM and TM mode components, at source-receiver offsets of 3, 7, and $11 \mathrm{~km}$ for the model of Figs 2 and 3. Fig. 4(b) contains the Fréchet derivatives with respect to the vertical conductivity at the same offsets; these are entirely a TM mode phenomenon. The vertical conductivity derivatives show preferential sensitivity to conductivity changes within the resistive reservoir layer, and dominate the horizontal conductivity derivatives except at a $3 \mathrm{~km}$ offset below the reservoir layer. However, the phase of the vertical conductivity derivatives in the reservoir are such that an increase in conductivity results in an approximate decrease in the seafloor electric field at 3 and $7 \mathrm{~km}$ offset, but is nearly in quadrature at the longest offset. There is a large change in phase across the reservoir layer for the horizontal conductivity TM mode such that an increase in conductivity at shallow depths results in an approximate decrease in the in-line electric field, but an increase in conductivity at greater depths has 

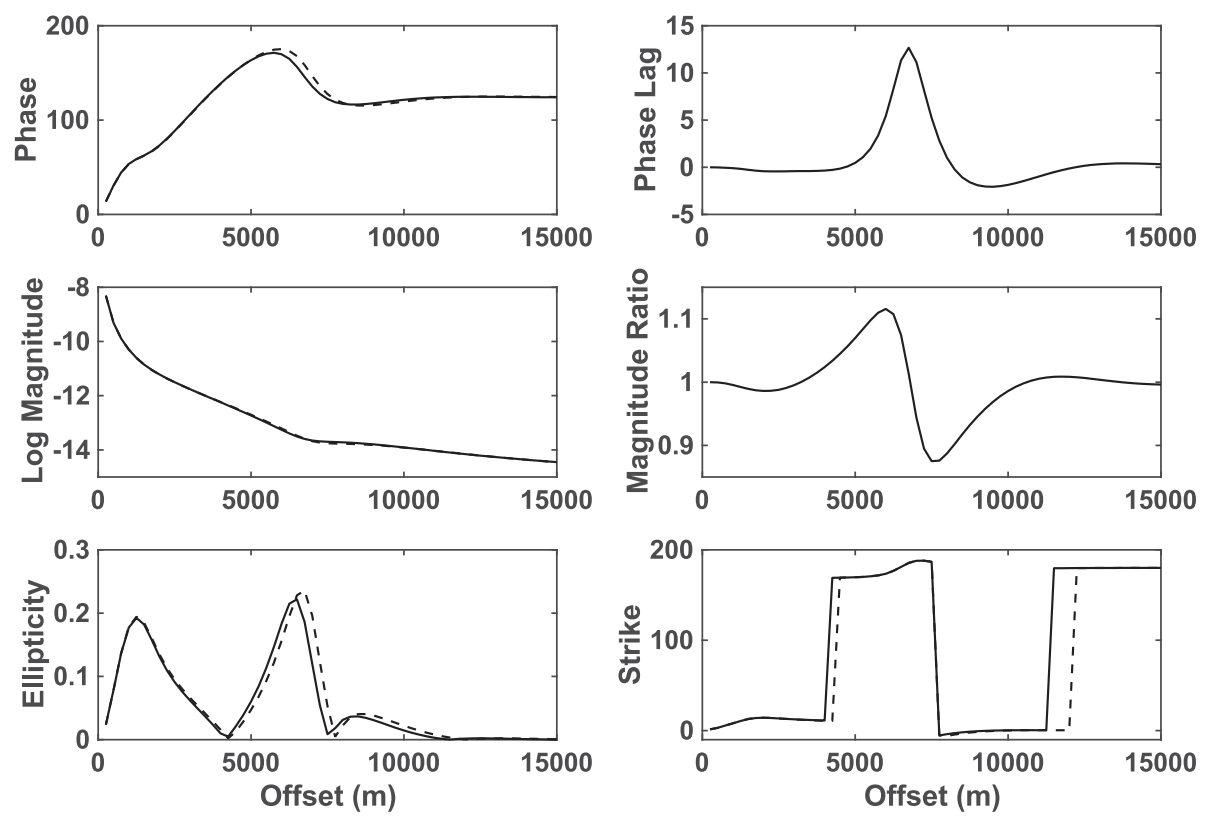

Figure 6. The polarization ellipse representation of the seafloor electric field as a function of offset from an HED point source placed at the origin but $30 \mathrm{~m}$ above the seafloor. The water depth is $300 \mathrm{~m}$ and the source frequency is $1 \mathrm{~Hz}$. From the upper left and proceeding counter-clockwise, the panels show the phase of the reservoir and halfspace responses, the base 10 logarithm of the magnitude of the reservoir and halfspace responses, the ellipticity, the strike of the semi-major axis in the ellipse plane, the magnitude ratio of the reservoir to the halfspace response and the phase lag between the reservoir and halfspace responses. For the first four parameters, the dashed line is the reservoir result while the solid line is the halfspace result.

the reverse effect. However, this behavior has only a limited effect given the dominance of the vertical conductivity derivatives.

Fig. 5 shows the Poynting vector and Joule heating for a water depth of $300 \mathrm{~m}$ and a $1 \mathrm{~Hz}$ source; the electrical thickness is 0.93 skin depth in the water layer and 0.82 skin depth in the overburden, so the air interaction is expected to play a comparable role as at $0.1 \mathrm{~Hz}$. The air interaction is manifest by downward energy flux within the water layer and its reversal at the seafloor from upward to downward at $\sim 5.5 \mathrm{~km}$ offset. The energy flux within the reservoir layer is outward to $\sim 12 \mathrm{~km}$ offset, and then rotates downward. By contrast, for an isotropic conductivity model, Fig. 12 of Paper I shows the rotation from upward to downward flux at the seafloor at $\sim 3 \mathrm{~km}$, and outward energy flux in the reservoir layer to $\sim 6 \mathrm{~km}$ offset followed by gradual rotation to downward. The Joule heating in Fig. 5 is nearly independent of depth below the seafloor, and is quite different from the isotropic result, where the reservoir layer is either a persistent locus of enhanced or decreased dissipation.

Fig. 6 shows the seafloor electric field for the $300 \mathrm{~m}, 1 \mathrm{~Hz}$ transversely anisotropic model. The amplitude ratio is very slightly below unity over $\sim 1-3 \mathrm{~km}$, larger than that value over $\sim 3-6 \mathrm{~km}$, then below unity over $\sim 6-10 \mathrm{~km}$, and finally very slightly above 1 beyond that point. The phase lag is positive from $\sim 5$ to $8 \mathrm{~km}$ offset, then slightly negative out to $\sim 11 \mathrm{~km}$. Consequently, the response is stronger and slower over $\sim 5-7 \mathrm{~km}$, then weaker and slower over $\sim 7-8 \mathrm{~km}$, and finally weaker and faster over $\sim 8-11 \mathrm{~km}$ offset. The polarization ellipse orientation is only slightly different for the reservoir and halfspace models. Overall, Fig. 6 resembles Fig. 13 of Paper I stretched to greater offsets.

Fig. 7(a) shows the in-line electric field Fréchet total derivatives, and its PM and TM mode components, for the horizontal conductivity and $1 \mathrm{~Hz}$ model. Fig. 7(b) shows the vertical conductivity derivatives. The vertical conductivity derivatives are dominant within the reservoir layer at all offsets, but have a similar magnitude to the horizontal conductivity derivatives in the underburden, and in the overburden at $11 \mathrm{~km}$ offset. The phase is approximately in quadrature within the reservoir layer for the vertical derivatives. The PM mode component of the horizontal conductivity derivatives is larger than the TM mode component within the overburden at $7 \mathrm{~km}$ and beyond, reflecting the increasing importance of the air interaction.

Fig. 8 shows the Poynting vector and Joule heating for shallow $(50 \mathrm{~m})$ water and a $0.1 \mathrm{~Hz}$ source. The water layer has an electrical thickness of about 0.06 skin depth compared to 0.26 skin depth in the overburden, and hence the air interaction is expected to be important at most offsets. The Poynting vector plot is qualitatively similar to that in Fig. 9 in Paper I, except that changes in direction occur at larger offsets throughout the structure. The direction of energy flux in the reservoir layer is fully reversed at offsets beyond $\sim 10 \mathrm{~km}$ in Fig. 8 compared to $\sim 5.5 \mathrm{~km}$ in Fig. 9 of Paper I. A minimum in the Poynting vector amplitude is also observed in Fig. 8 extending from near the source downward to the reservoir layer at $\sim 10 \mathrm{~km}$ and then further into the underburden. The locus of the minimum represents a shift from dominance by the dipole source (including galvanic reservoir excitation) to dominance by the air interaction. The outward energy flux tongue in the reservoir layer is nearly constant in length up to $\sim 10 \mathrm{~km}$, then increases over the interval exhibiting reversed energy flux. Joule heating is largest in the water layer at all offsets. In contrast to Fig. 9 in Paper I, the reservoir layer is a locus of only slightly enhanced dissipation.

Fig. 9 shows the seafloor electric field for the shallow water model of Fig. 8. The amplitude ratio is greater than unity beyond $\sim 2 \mathrm{~km}$ offset. The phase of the reservoir model weakly lags that for a halfspace over $\sim 3-8 \mathrm{~km}$, and has a stronger lead at larger offsets. Consequently, the reservoir response is stronger and slightly faster at short $(\sim 3-8 \mathrm{~km})$ offsets, and stronger and slower at longer offsets. The polarization is nearly linear at all offsets. The strike of the semi-major axis shifts counter-clockwise through $\sim 180^{\circ}$ at 

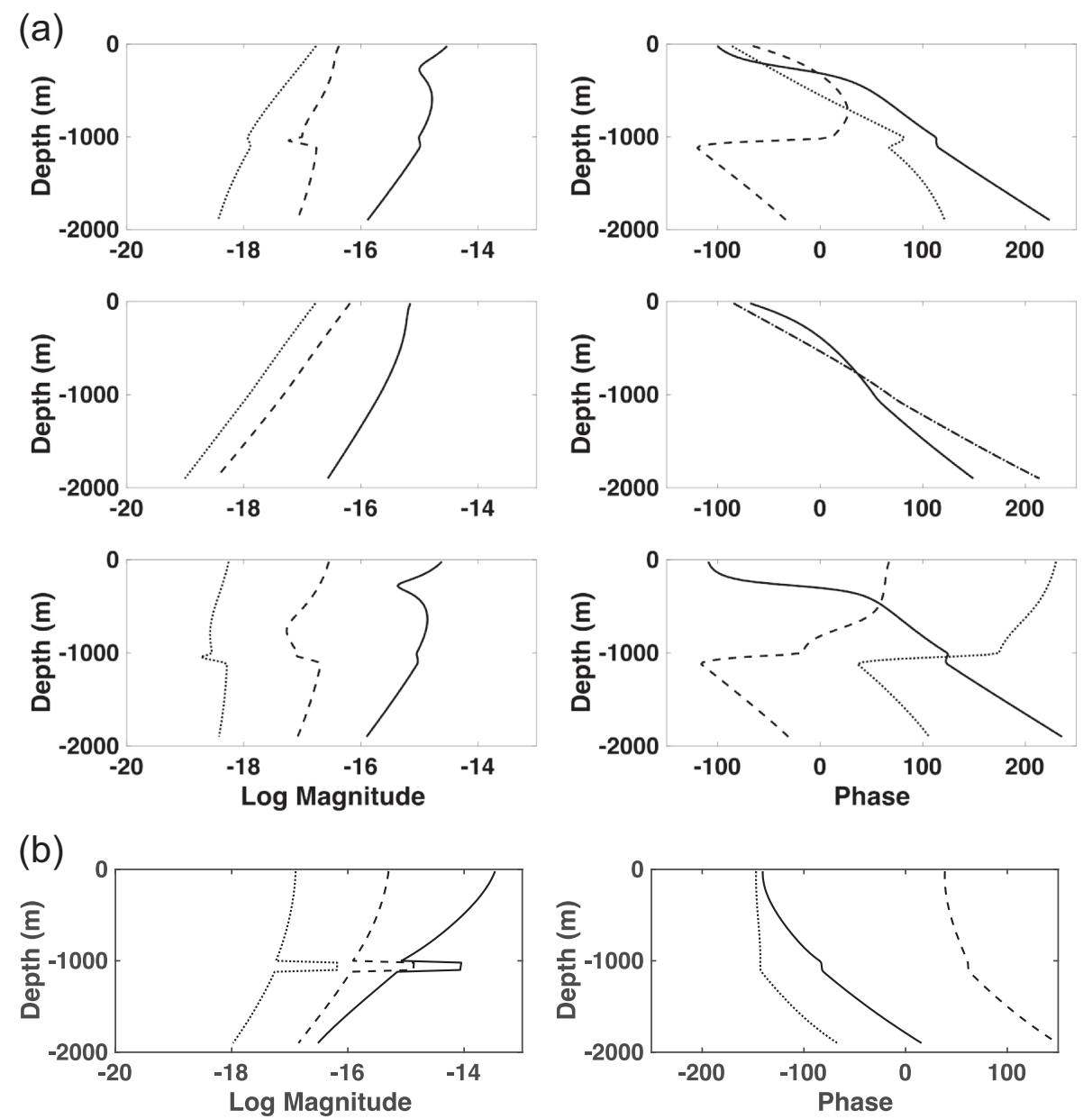

Figure 7. The Fréchet derivatives with respect to the horizontal conductivity (a) and vertical conductivity (b) for the in-line seafloor electric field as a function of depth below the seafloor for a point HED source located at the origin but $30 \mathrm{~m}$ above the seafloor. The water depth is $300 \mathrm{~m}$ and the source frequency is $1 \mathrm{~Hz}$. The left panels show the logarithm of the magnitude and the right panels show the phase. From top to bottom, the rows in (a) show the total Fréchet derivative, the PM mode component and the TM mode component. The different curves correspond to offsets of $3 \mathrm{~km}$ (black), $7 \mathrm{~km}$ (dashed) and $11 \mathrm{~km}$ (dotted).

$\sim 7.5 \mathrm{~km}$ offset for both models. Overall, Fig. 9 resembles Fig. 10 in Paper I stretched to longer offsets.

Fig. 10(a) shows the in-line electric field Fréchet total derivative, and the PM and TM mode components, for the horizontal conductivity of the $50 \mathrm{~m}$ water depth, $0.1 \mathrm{~Hz}$ model. Fig. 10(b) shows the vertical conductivity derivatives. Preferential sensitivity to the vertical conductivity is dominant at all offsets within the reservoir layer. The horizontal and vertical derivatives are comparable within the substrate at the shortest offset, but the vertical derivative is larger at the longest ones.

Fig. 11 shows the Poynting vector and Joule heating for a 50-mthick water layer but with a $1 \mathrm{~Hz}$ source. At this frequency, the water layer is 0.18 skin depth thick, whereas the overburden is 0.82 skin depths thick, and hence the reservoir effect should be dwarfed by the influence of the sea surface beyond some offset. By comparison with Fig. 8, the magnitudes of the Poynting vector and Joule heating are smaller at a given offset and depth, reflecting greater attenuation throughout the structure due to the higher frequency. The seafloor energy flux shifts from upward to downward, but with an inward component, at a source-receiver offset of $\sim 3.5 \mathrm{~km}$, and the flip in direction propagates downward to the reservoir layer at $\sim 6 \mathrm{~km}$. The time-averaged energy flux is downward throughout the structure beyond $\sim 11 \mathrm{~km}$, and the rightward tongue of enhanced energy flux vanishes. This reflects the fact that, at the higher frequency of $1 \mathrm{~Hz}$, the dipole field is heavily attenuated at long range, whereas the spatially broad field due to the air interaction does not decrease as much. Concomitantly, the reservoir layer is a local minimum of increased dissipation with offset.

Fig. 12 shows the seafloor electric field corresponding to Fig. 11, and resembles a compressed and attenuated version of Fig. 9. The magnitude ratio is nearly unity to $\sim 4 \mathrm{~km}$, then smaller than one to $\sim 8 \mathrm{~km}$ and then slightly larger than one to $\sim 11 \mathrm{~km}$. The phase leads that for a halfspace over $\sim 3-5 \mathrm{~km}$, then lags slightly to $\sim 10 \mathrm{~km}$. Consequently, the reservoir model response is initially weaker and slower $(\sim 4-5 \mathrm{~km})$, then weaker and faster $(\sim 5-8 \mathrm{~km})$, and finally stronger and faster $(\sim 8-10 \mathrm{~km})$, as compared to the halfspace response. The ellipticity has a peak at $\sim 4.5 \mathrm{~km}$, and is larger than at $0.1 \mathrm{~Hz}$ in Fig. 9. The strike changes repeatedly with offset, but is similar over $0-5 \mathrm{~km}$ to the entire range of Fig. 9 .

Fig. 13(a) shows the in-line electric field Fréchet total derivative, and its PM and TM mode components, for the horizontal conductivity and $1 \mathrm{~Hz}$ model. Fig. 13(b) shows the vertical conductivity derivatives. The vertical conductivity derivatives are dominant within the reservoir layer at all offsets, but the picture in the underburden and overburden is complex. At $3 \mathrm{~km}$ offset, sensitivity to the vertical conductivity overwhelms that to the horizontal resistivity in 

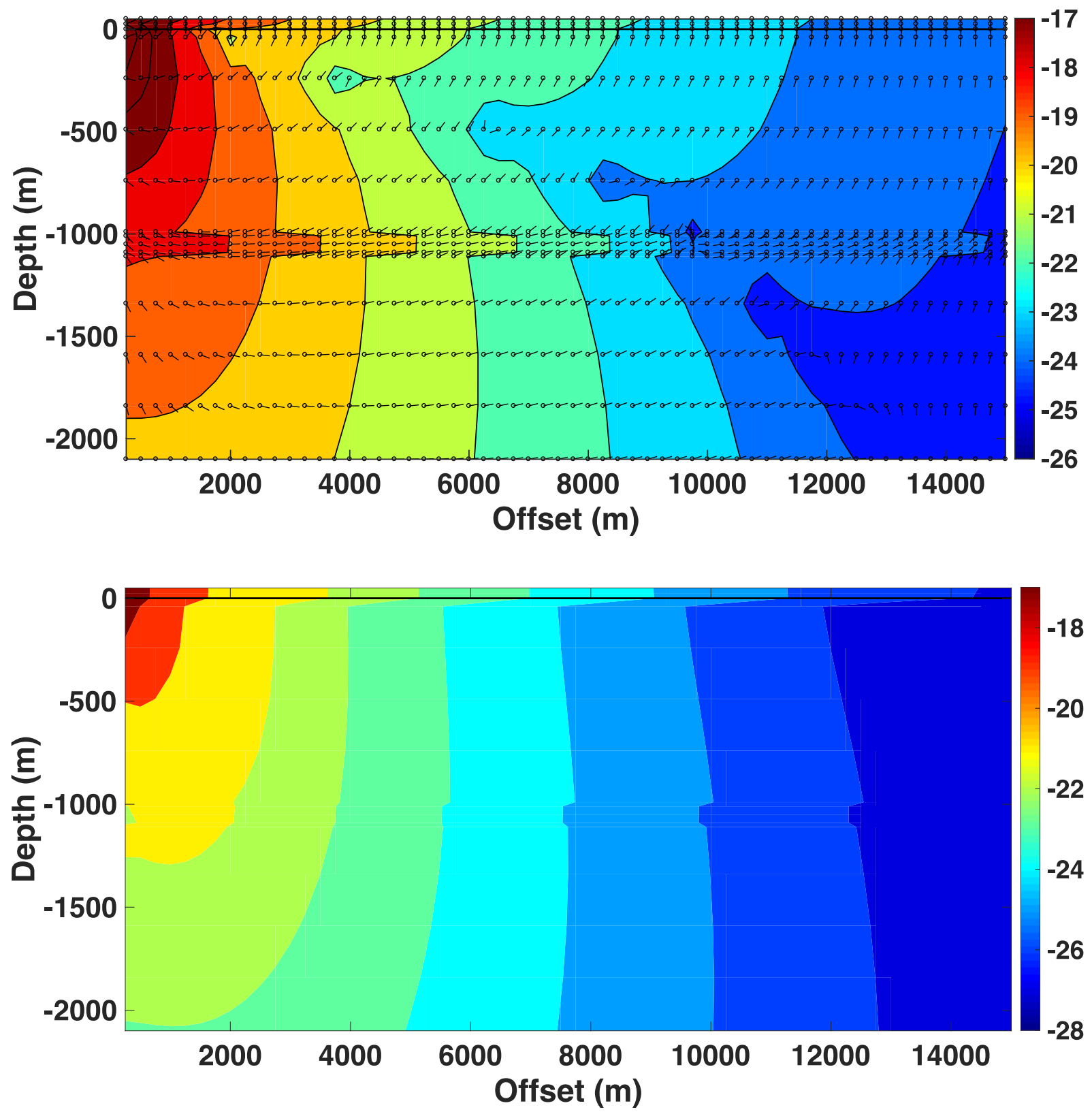

Figure 8. Contours of the logarithms (base 10) of the magnitudes of the Poynting vector (top) and Joule heating (bottom) as a function of source-receiver offset and depth for the model of Fig. 1. The water depth is $50 \mathrm{~m}$, the source frequency is $0.1 \mathrm{~Hz}$ and the point source lies $30 \mathrm{~m}$ above the seafloor at the origin. The seafloor is depicted by a solid horizontal black line. The Poynting vector plot also shows the direction of energy flow at each of the small circles throughout the structure. The arrow orientations have been adjusted for the different horizontal and vertical scales.

the overburden, whereas the vertical and horizontal derivatives are comparable in the underburden. At $7 \mathrm{~km}$ offset, sensitivity to the vertical conductivity is larger except near the seafloor. At $11 \mathrm{~km}$, sensitivity to the horizontal conductivity is dominant in the overburden, whereas the vertical and horizontal derivatives are comparable in the underburden. These differences reflect the presence of a PM mode component that is comparable in size to the TM mode, leading to a complex interplay of the two types of Fréchet derivatives.

The Poynting vector for $300 \mathrm{~m}$ water depth at $0.1 \mathrm{~Hz}$ in the shallow towed configuration is very similar to Fig. 2. Fig. 14 shows the Poynting vector for $300 \mathrm{~m}$ water depth and a $1 \mathrm{~Hz}$ source in the shallow towed configuration. It is very similar to the seafloor configuration result in Fig. 5, with the key feature of transition from upward to downward energy flux at the seafloor, and its propagation through the overburden, occurring at a $\sim 2 \mathrm{~km}$ shorter offset. The Joule heating for the shallow towed model is nearly identical to that in Fig. 5, and is omitted.

It is not practical to measure the vertical electric field from a towed streamer, hence Fig. 15 shows the in-line electric field parameters corresponding approximately to the four upper panels in Fig. 6. The shallow towed electric field is quite different from the seafloor one for this model, displaying an initial stronger and very slightly faster reservoir response from $\sim 1$ to $3 \mathrm{~km}$ offset, then a stronger and slower response to $\sim 5 \mathrm{~km}$, a weaker and faster response to $\sim 6 \mathrm{~km}$, a stronger and faster response to $\sim 8 \mathrm{~km}$, a stronger and slower response to $\sim 10 \mathrm{~km}$ and finally a weaker and slower response 

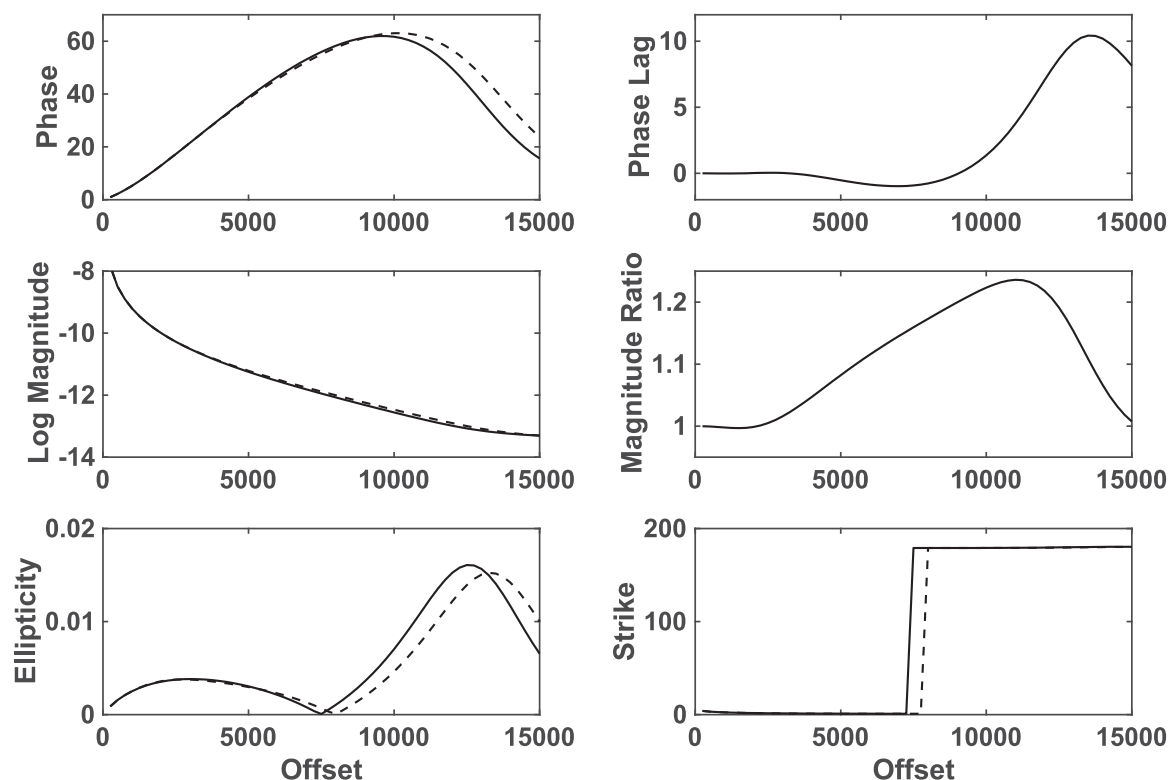

Figure 9. The polarization ellipse representation of the seafloor electric field as a function of offset from an HED point source placed at the origin but $30 \mathrm{~m}$ above the seafloor. The water depth is $50 \mathrm{~m}$ and the source frequency is $0.1 \mathrm{~Hz}$. From the upper left and proceeding counter-clockwise, the panels show the phase of the reservoir and halfspace responses, the base 10 logarithm of the magnitude of the reservoir and halfspace responses, the ellipticity, the strike of the semi-major axis in the ellipse plane, magnitude ratio of the reservoir to the halfspace response and the phase lag between the reservoir and halfspace responses. For the first four parameters, the dashed line is the reservoir result whereas the solid line is the halfspace result.
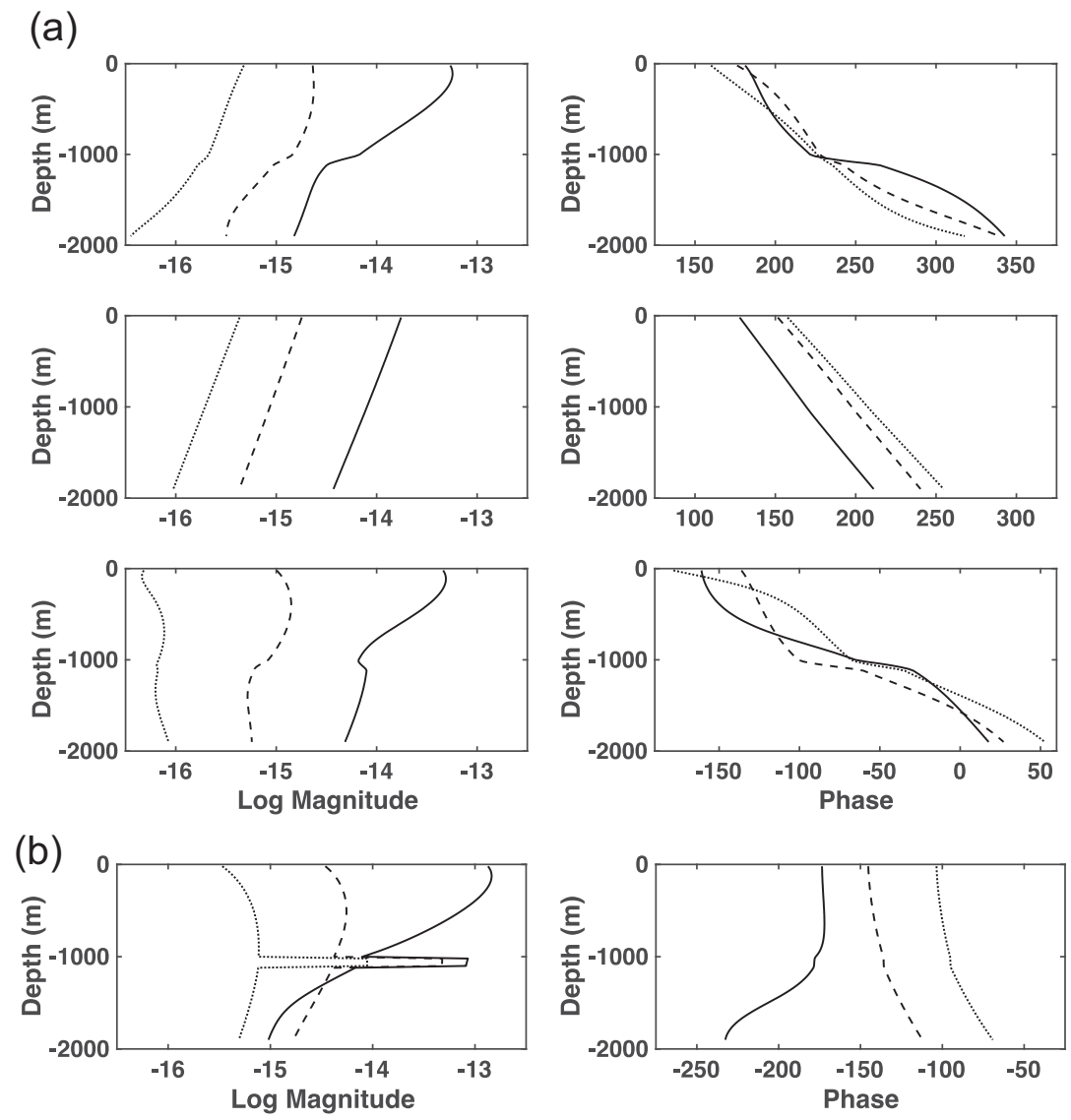

Figure 10. The Fréchet derivatives with respect to the horizontal conductivity (a) and vertical conductivity (b) for the in-line seafloor electric field as a function of depth below the seafloor for a point HED source located at the origin but $30 \mathrm{~m}$ above the seafloor. The water depth is $50 \mathrm{~m}$ and the source frequency is $0.1 \mathrm{~Hz}$. The left-hand panels show the logarithm of the magnitude and the right-hand panels show the phase. From top to bottom, the rows in (a) show the total Fréchet derivative, the PM mode component and the TM mode component. The different curves correspond to offsets of $3 \mathrm{~km}$ (black), $7 \mathrm{~km}$ (dashed) and $11 \mathrm{~km}$ (dotted). 

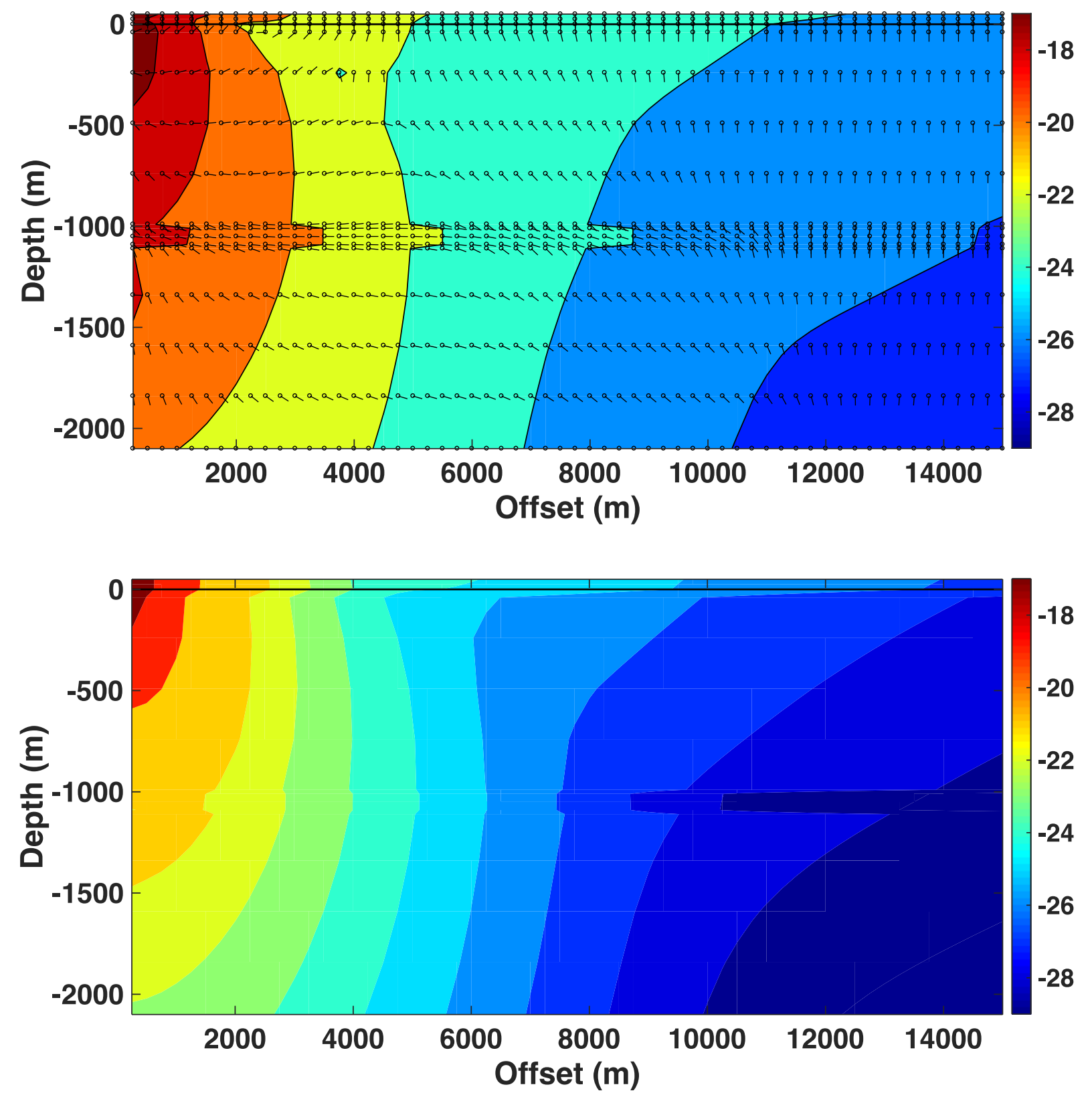

Figure 11. Contours of the logarithms (base 10) of the magnitudes of the Poynting vector (top) and Joule heating (bottom) as a function of source-receiver offset and depth for the model of Fig. 1. The water depth is $50 \mathrm{~m}$, the source frequency is $1 \mathrm{~Hz}$ and the point source lies $30 \mathrm{~m}$ above the seafloor at the origin. The seafloor is depicted by a solid horizontal black line. The Poynting vector plot also shows the direction of energy flow at each of the small circles throughout the structure. The arrow orientations have been adjusted for the different horizontal and vertical scales.

to $\sim 12 \mathrm{~km}$. The differences between the reservoir and halfspace responses in Fig. 15 are quite small.

Fig. 16 shows the Fréchet derivatives for the shallow towed configuration model of Figs 14 and 15. It is only subtly distinct from Fig. 7, and displays a similar complexity with offset. The vertical Fréchet derivative is dominant within the reservoir layer at all offsets. At $3 \mathrm{~km}$ offset, sensitivity to the vertical conductivity is larger than to the horizontal component to $\sim 800 \mathrm{~m}$ depth in the overburden, below which the horizontal conductivity derivative is increasingly larger through the underburden. At $7 \mathrm{~km}$ offset, the vertical conductivity derivative dwarfs the horizontal one throughout the substrate. At $11 \mathrm{~km}$ offset, the vertical and horizontal derivatives are comparable though out the substrate. This can be understood through the interplay between the PM and TM modes in Fig. 16(a).
The horizontal conductivity TM mode is much larger than the PM mode one at $3 \mathrm{~km}$, but the PM mode is increasingly important with offset.

For $50 \mathrm{~m}$ water depth at either 0.1 or $1 \mathrm{~Hz}$, the shallow towed configuration responses are quite similar to the seafloor ones in Figs 8-13, and are omitted.

\section{DISCUSSION}

The Poynting vector is proportional to the cross product of the electric and magnetic fields, and hence is a second order quantity. The PM and TM mode elements of the electric and magnetic fields are independent as a matter of physics, but this independence does 

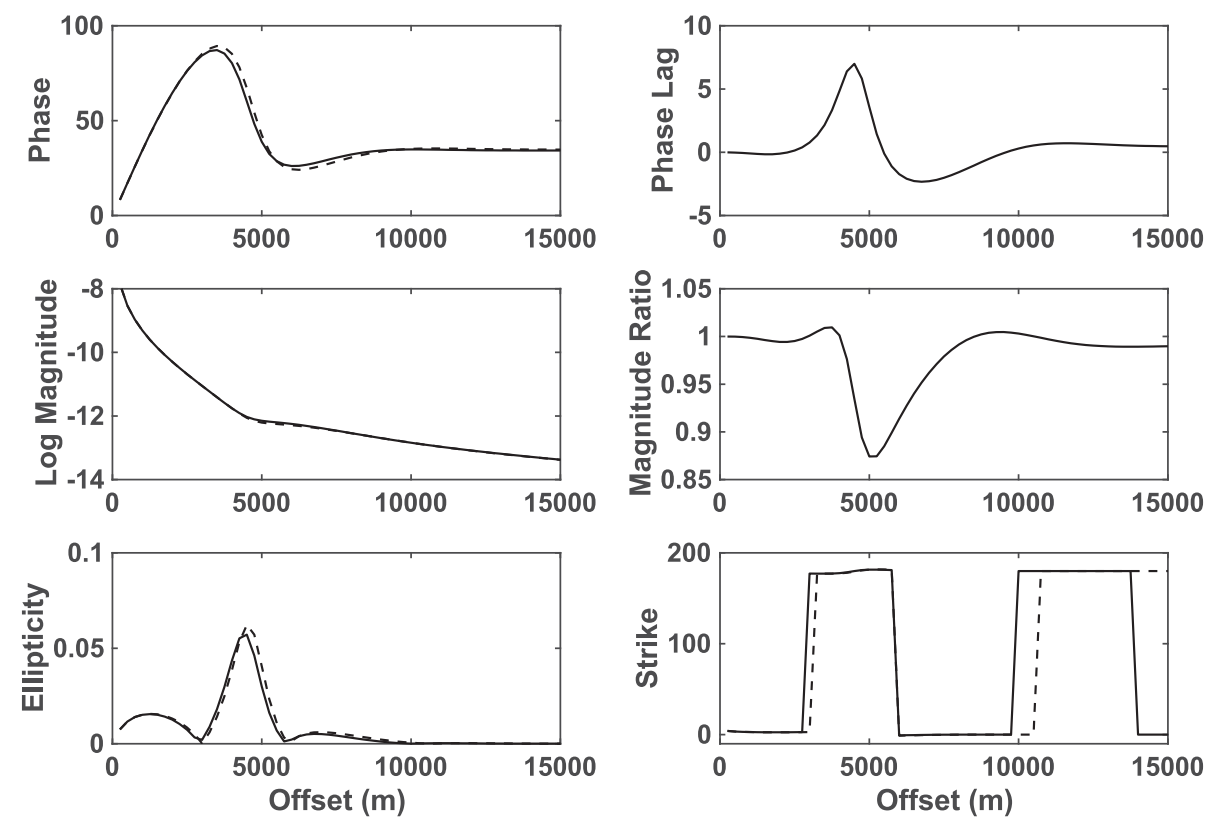

Figure 12. The polarization ellipse representation of the seafloor electric field as a function of offset from an HED point source placed at the origin but $30 \mathrm{~m}$ above the seafloor. The water depth is $50 \mathrm{~m}$ and the source frequency is $1 \mathrm{~Hz}$. From the upper left and proceeding counter-clockwise, the panels show the phase of the reservoir and halfspace responses, the base 10 logarithm of the magnitude of the reservoir and halfspace responses, the ellipticity, the strike of the semi-major axis in the ellipse plane, magnitude ratio of the reservoir to the halfspace response and the phase lag between the reservoir and halfspace responses. For the first four parameters, the dashed line is the reservoir result whereas the solid line is the halfspace result.

not pertain for the Poynting vector. The time-averaged energy flux is given by the complex Poynting vector

$\boldsymbol{S}=\frac{\left[\left(\mathbf{E}_{P M}+\mathbf{E}_{T M}\right) \times\left(\mathbf{B}_{P M}^{*}+\mathbf{B}_{T M}^{*}\right)\right]}{2 \mu_{\mathrm{o}}}$.

When expanded, (5) comprises purely PM and TM contributions, along with two cross terms that couple the PM and TM mode components in the electric and magnetic fields. For a source that produces only a PM mode (vertical magnetic dipole) or only a TM mode (vertical electric dipole), Fig. 11 in Chave (2009) shows that the former includes an air interaction whereas the latter is insensitive to the presence of the sea surface. However, the Poynting vector for an HED source cannot be described as a superposition of these two end members for either an isotropic or anisotropic substrate.

The Poynting vector results in Figs 2, 5, 8, 11, and 14 reflect the competing effects of the dipole source (including galvanic, guided energy flow within the reservoir) and the inductive air interaction, along with coupling of the two EM modes as in (5). For the seafloor configuration, when the ocean is of comparable electrical thickness to the overburden (Figs 2 and 5), the air interaction does not appear except at long offsets. When the electrical thickness of the water layer is smaller than that of the overburden (Figs 8 and 11), the dipole source and guided energy flow are predominant at short offsets, and the air interaction is larger at long offsets, yielding a transition zone that moves from the water layer through the overburden and into the underburden. Under some circumstances (Fig. 8), the cross-mode terms in (5) are polarized appropriately, and strong enough, at the reservoir layer to reverse the time-averaged energy flux direction at long offsets. This phenomenon cannot be solely due to either the PM or TM modes, as witnessed by Fig. 11 in Chave (2009). For the shallow towed configuration, Fig. 14 displays similar flux reversal behavior to the seafloor configuration, but with a shift of transition to slightly shorter offsets.
The Joule heating can easily be understood in terms of continuity of vertical electric current versus continuity of the in-line electric field at the reservoir layer boundaries. For deeper water and low frequencies (Fig. 2), the electric field is nearly vertical within the reservoir layer at all ranges. Consequently, by continuity of vertical electric current, the vertical electric field in the reservoir must be about a factor of 3 in magnitude larger than in the overburden or underburden because the reservoir conductivity is $1 / 3$ that in those regions, implying that Joule heating is always slightly enhanced within the reservoir layer at a given offset. However, as the air interaction predominates over the galvanic reservoir response (Figs 8 and 11), the electric field becomes increasingly radial, and hence will have the same value in the reservoir layer and substrate, resulting in reduced Joule heating because the reservoir conductivity is lower than in its surroundings. The contrasts in this paper are more modest than in Paper I because the vertical resistivity ratio is $3: 1$ rather than 10:1 at the reservoir layer.

When expressed as the ratio of the magnitude of the reservoir response to that of a halfspace with the overburden resistivity, and the phase lag between the reservoir and the halfspace model, both as a function of source-receiver offset, the amplitude ratio consistently peaks where the phase lag is changing most rapidly. Consequently, a phase response to a structure is always observed at shorter ranges than an amplitude response. This is clearly observed in the electric field for Figs 3, 6, 9, 12 and 15, and was also seen in Paper I.

The behaviors of the seafloor electric and magnetic fields are tightly associated with energy flow through the entire conductive structure. Fig. 2 shows no effect of the downward propagating energy in the water layer and overburden within the reservoir layer. However, the seafloor electric field in Fig. 3 clearly reflects its presence beyond $\sim 13 \mathrm{~km}$ offset as the phase lag begins to decrease. This is more apparent in Figs 5 and 6, where the air interaction is increasingly dominant within the overburden beyond $\sim 10 \mathrm{~km}$. An initial 
(a)
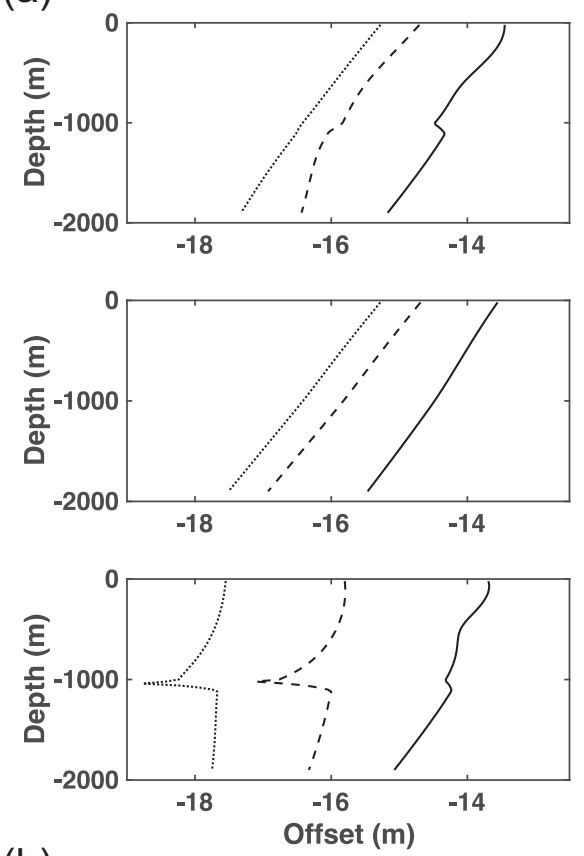

(b)

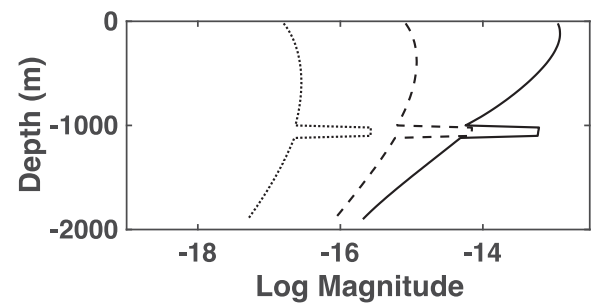

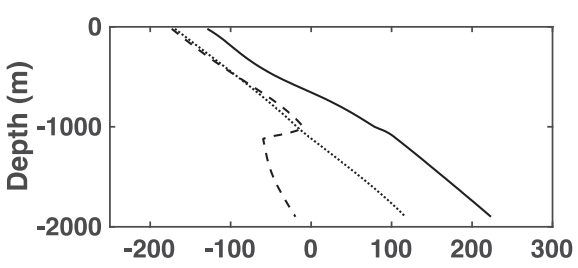
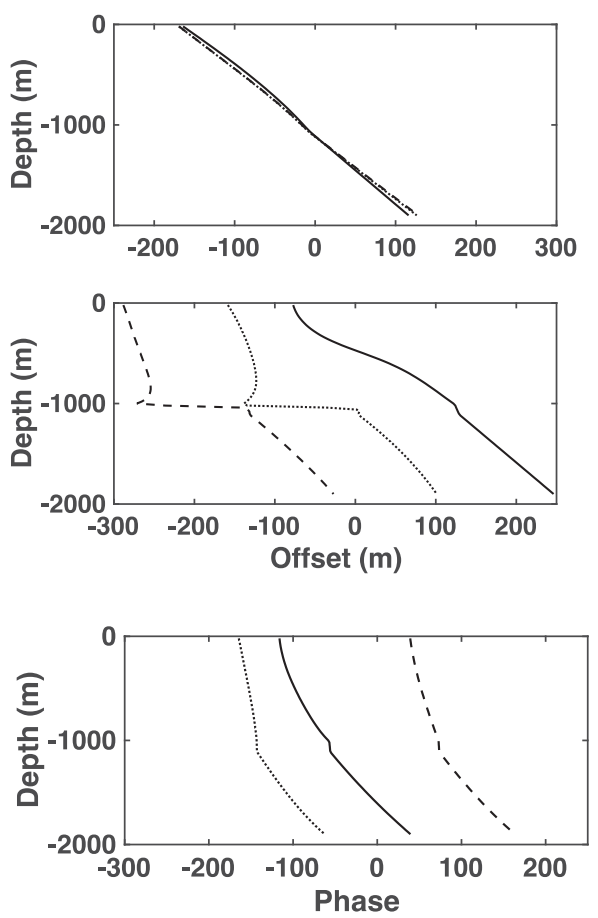

Figure 13. The Fréchet derivatives with respect to the horizontal conductivity (a) and vertical conductivity (b) for the in-line seafloor electric field as a function of depth below the seafloor for a point HED source located at the origin but $30 \mathrm{~m}$ above the seafloor. The water depth is $50 \mathrm{~m}$ and the source frequency is $1 \mathrm{~Hz}$. The left-hand panels show the logarithm of the magnitude and the right-hand panels show the phase. From top to bottom, the rows in (a) show the total Fréchet derivative, the PM mode component and the TM mode component. The different curves correspond to offsets of $3 \mathrm{~km}$ (black), $7 \mathrm{~km}$ (dashed) and $11 \mathrm{~km}$ (dotted).

phase lead for the reservoir model reflects the influence of guided energy flow in the reservoir layer that is increasingly counteracted by downward energy flux from the air interaction that is associated with a phase lag; the direction of energy flow at the seafloor shifts from upward to downward approximately at the inflection point in the phase. This is also apparent in Figs 14 and 15. The key difference from the Paper I results is that the changes in magnitude ratio and phase lag shift to longer offsets in the presence of transverse anisotropy.

When the ocean is electrically much thinner than the overburden, Figs 8, 9, 11 and 12 show a similar effect at smaller sourcereceiver offsets, and in addition display a more substantial slow response due to a Poynting vector that has a component directed back at the source. This is especially strong in Figs 8 and 9, where the energy flux in the reservoir layer is reversed beyond $\sim 10 \mathrm{~km}$ offset.

As in Paper I, the results of this paper show that CSEM in intermediate to shallow water remains sensitive to the substrate conductivity. This is most easily seen through the Fréchet derivatives in Figs 4, 7, 10, 13 and 16. A key distinction between the isotropic case in Paper I and the present one is that there is preferential sensitivity to the vertical resistivity within the reservoir layer for all cases that are described. There is also typically preferred sensitivity to the ver- tical resistivity in the overburden at short offsets. Beyond, these two points, the Fréchet derivatives display a complex interplay between the TM and PM modes for the horizontal conductivity, and an additional interplay between the horizontal and vertical conductivity derivatives, as described in Section 3. In general, it is necessary to move to higher frequencies in the presence of transverse anisotropy to detect the horizontal conductivity in the substrate, as is apparent from comparing Figs 7, 13 and 16 to 5 and 10.

Consequently, the air interaction enables the detection of both anisotropy and a reservoir layer with only the in-line geometry if the water is not too deep, providing that the experimental parameters (i.e. source frequency and source-receiver offset) are chosen appropriately. The need to shift to higher frequencies to resolve the overburden conductivity underscores the importance of presurvey modeling studies to choose optimal offsets and frequencies for candidate structures.

\section{CONCLUSIONS}

This paper has presented a study of the physics of marine CSEM in shallow water for a resistive oil reservoir layer embedded within a substrate that is transversely anisotropic, with a vertical-tohorizontal resistivity ratio of $3: 1$. Because of the electrical thinness 


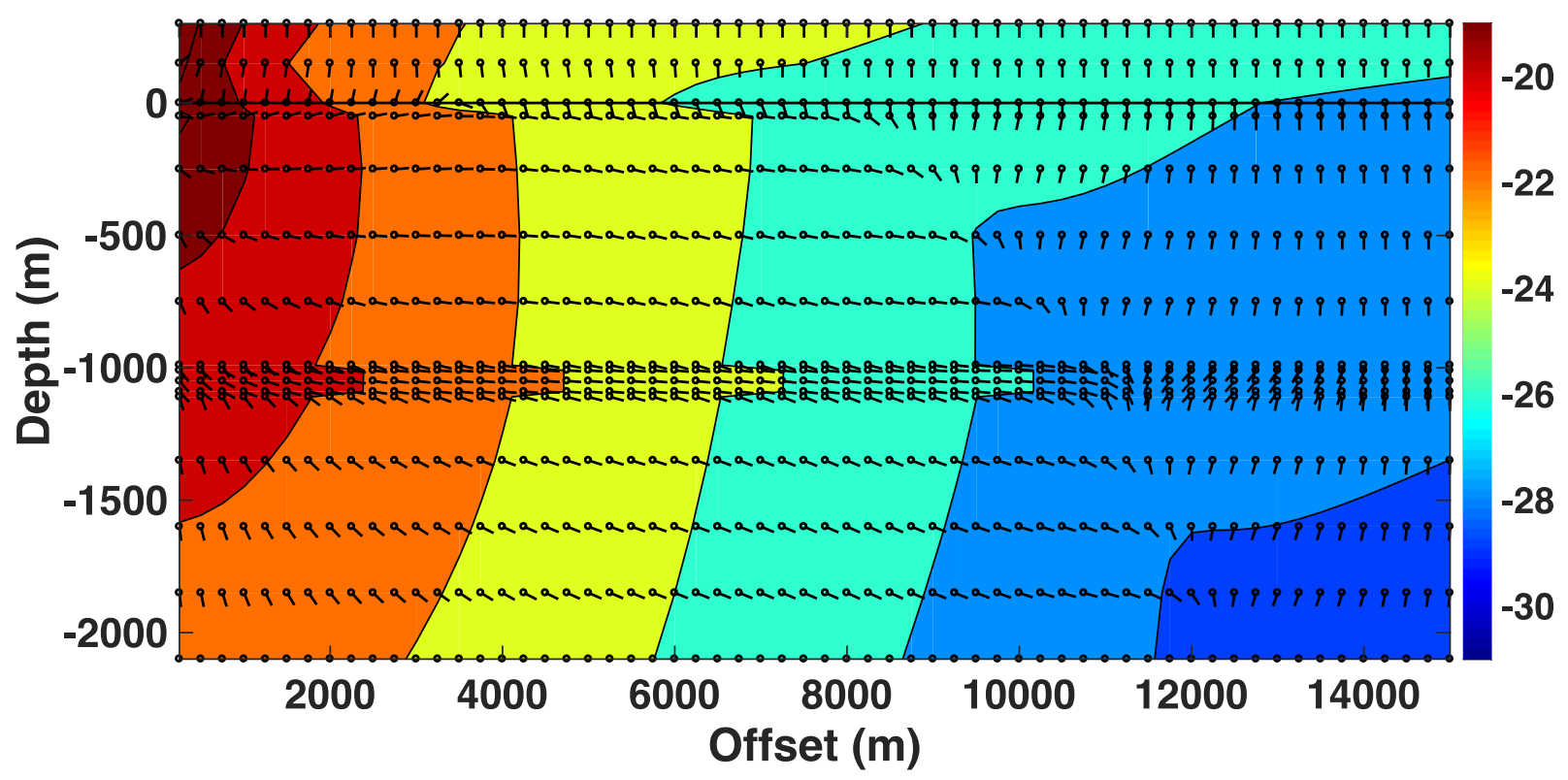

Figure 14. Contours of the logarithm (base 10) of the magnitude of the Poynting vector as a function of source-receiver offset and depth for the model of Fig. 1. The water depth is $300 \mathrm{~m}$, the source frequency is $1 \mathrm{~Hz}$ and the point source lies $10 \mathrm{~m}$ below the sea surface at the origin. The receivers lie at $100 \mathrm{~m}$ water depth in the shallow towed configuration. The seafloor is depicted by a solid horizontal black line. The Pointing vector plot also shows the direction of energy flow at each of the small circles throughout the structure. The arrow orientations have been adjusted for the different horizontal and vertical scales.
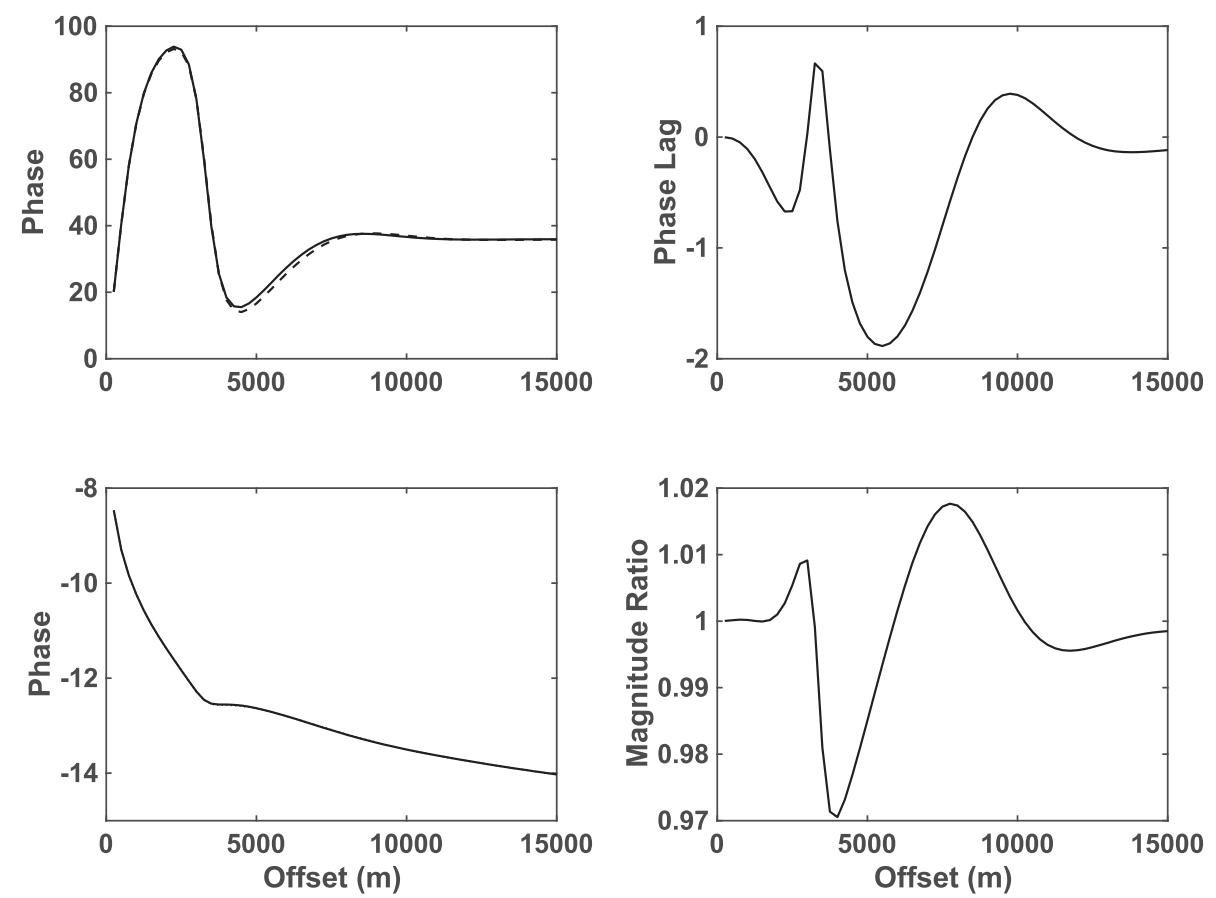

Figure 15. The in-line electric field at $100 \mathrm{~m}$ water depth as a function of offset from an HED point source placed at the origin but $10 \mathrm{~m}$ below the sea surface. The water depth is $300 \mathrm{~m}$ and the source frequency is $1 \mathrm{~Hz}$. From the upper left and proceeding counter-clockwise, the panels show the phase of the reservoir and halfspace responses, the base 10 logarithm of the magnitude of the reservoir and halfspace responses, the magnitude ratio of the reservoir to the halfspace response and the phase lag between the reservoir and halfspace responses. The dashed line is the reservoir result whereas the solid line is the halfspace result.

of the water layer, the seafloor electric field responds in a complicated way through the combination of a dipole field with guided energy flow within the hydrocarbon layer and an air interaction that exists throughout the subsurface structure due to the presence of the insulating atmosphere above the ocean. The additional degree of freedom provided by separate vertical and horizontal compo- nents of conductivity results in further complexity compared to the isotropic case.

When expressed as the ratio of the magnitude of the reservoir response to that of a halfspace with the overburden resistivity, and the phase lag between the reservoir and halfspace models, both as a function of source-receiver offset, the amplitude ratio consistently 
(a)
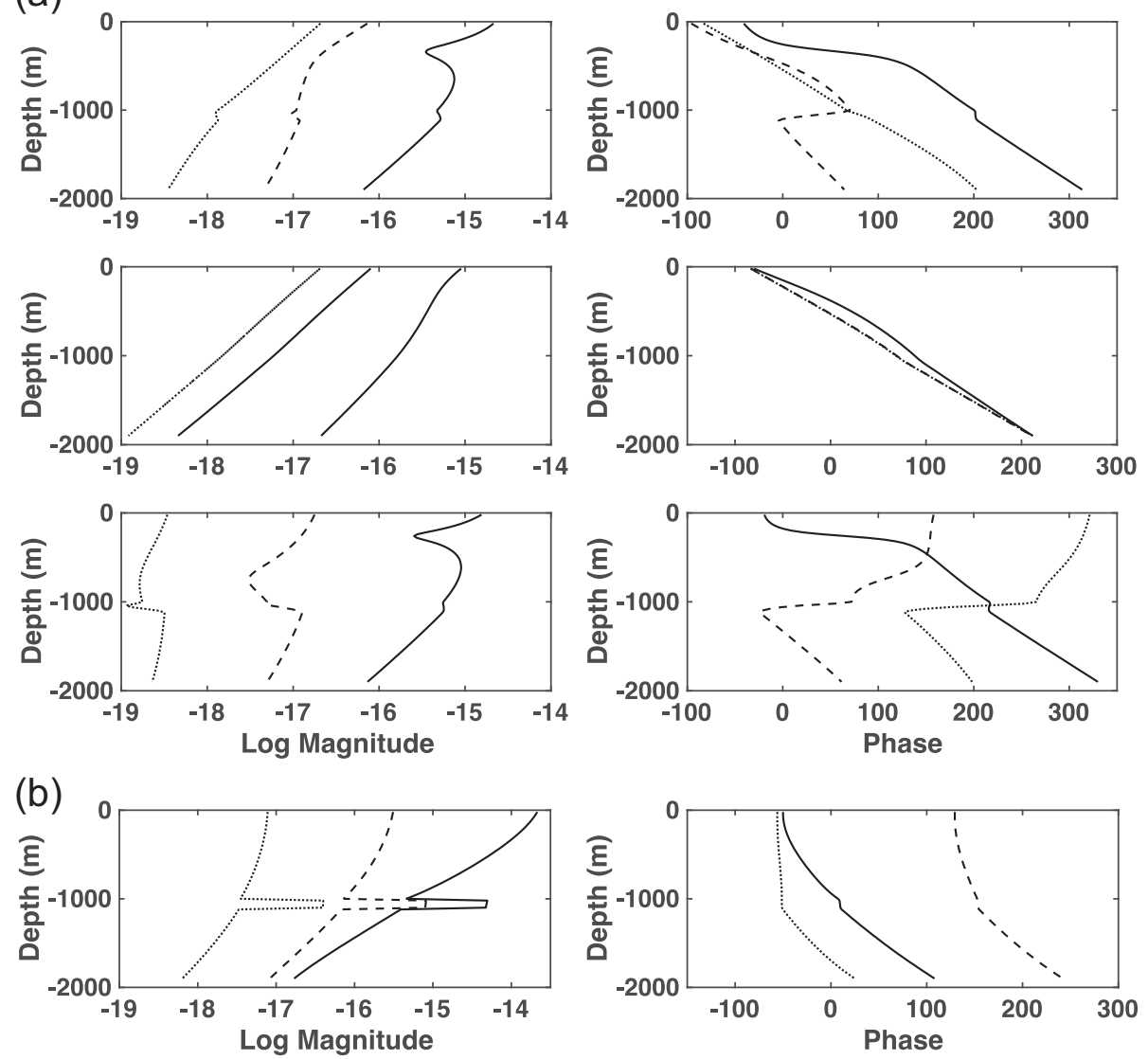

Figure 16. The Fréchet derivatives with respect to the horizontal conductivity (a) and vertical conductivity (b) for the in-line seafloor electric field as a function of depth below the seafloor for a point HED source located at the origin but $10 \mathrm{~m}$ below the sea surface, and receivers towed at $100 \mathrm{~m}$ depth. The water depth is $300 \mathrm{~m}$ and the source frequency is $1 \mathrm{~Hz}$. The left-hand panels show the logarithm of the magnitude and the right-hand panels show the phase. From top to bottom, the rows in (a) show the total Fréchet derivative, the PM mode component and the TM mode component. The different curves correspond to offsets of $3 \mathrm{~km}$ (black), $7 \mathrm{~km}$ (dashed) and $11 \mathrm{~km}$ (dotted).

peaks where the phase lag is changing most rapidly. Consequently, a phase response to a structure is always observed at shorter offsets than an amplitude response. This observation pertains equally to an isotropic substrate.

When the electrical thickness of the ocean becomes comparable to that of the overburden, a general picture of a stronger and faster reservoir model response compared to that for a transversely anisotropic halfspace is observed. This result is quite similar to the deep water response for an isotropic substrate. When the electrical thickness of the water layer is substantially smaller than that of the overburden, the same pattern of a stronger and faster response, followed by a stronger and slower one and finally a weaker and slower response is sometimes observed, but additional permutations can exist. The general pattern in the presence of transverse anisotropy is a stretching of the response observed for an isotropic model with the same horizontal conductivity to longer offsets.

As for the isotropic case, the behavior of the seafloor electric field may be understood through visualization of the flow of energy throughout the structure. In intermediate water depths, guided energy flow in the reservoir layer dominates the Poynting vector, and the result closely resembles the deep water isotropic response at the same frequency. At small offsets in shallow water, guided energy flow in the resistive reservoir layer is dominant, resulting in an enhanced seafloor electric field and phase lead over that from a halfspace, or the stronger and faster response seen pervasively in deep water for an isotropic model. At large offsets, the air interaction is dominant, energy flow is downward throughout the structure and hence the response becomes weaker and slower. At intermediate offsets, a couple of factors come into play. First, a transition locus in energy flow from upward to downward propagates in the structure from the sea surface through the overburden as offset increases, causing marked changes in the seafloor electric field. Second, the complex interplay of the PM and TM modes in the second order Poynting vector (5) can actually cause the energy flow in the reservoir layer to shift inward toward the source to varying degrees over a limited set of ranges, enhancing the intermediate, observed stronger but slower response. These observations occur at larger offsets in the presence of transverse anisotropy than for the corresponding isotropic case.

Joule heating within a volume of material that balances the timeaveraged energy flux into it is enhanced within the reservoir layer when guided energy flow is important. However, when the air interaction is dominant, the reservoir layer becomes a local locus of minimum dissipation. These end member behaviors can be understood in terms of the boundary conditions on the vertical electric current and horizontal electric field at the reservoir layer. These observations pertain equally to the isotropic case, although the differences are more subdued in the presence of transverse anisotropy because the ratio of vertical substrate conductivity to that of the reservoir is smaller. 
The Fréchet derivatives of the seafloor electric field with respect to the vertical electrical conductivity pervasively show a strong peak in the reservoir layer that dominates at all offsets and frequencies. This in part explains why marine CSEM remains sensitive to a hydrocarbon layer as water depth decreases. For the horizontal conductivity, while the TM mode derivatives remain large in the underburden and overburden at short offsets and low frequencies, the inductive PM mode from the air interaction becomes increasingly dominant at longer offsets and higher frequencies. The interplay of the TM and PM modes for the horizontal derivatives, and of the vertical and horizontal derivatives, results in more complexity for the transverse anisotropic case as compared to the isotropic one. However, by sampling a suitable region of parameter (i.e. frequency and source-receiver offset) space, it is possible to measure both the horizontal and vertical components of conductivity in these regions using only the in-line geometry. This will typically require the use of higher frequencies in the presence of transverse anisotropy as compared to an isotropic model with the same horizontal conductivity.

\section{ACKNOWLEDGEMENTS}

This work was supported at WHOI by an Independent Research and Development award, and by the Walter A. and Hope Noyes Smith Chair for Excellence in Oceanography.

\section{REFERENCES}

Anderson, B., Bryant, I., Luling, M., Spies, B. \& Helbig, K., 1994. Oil-field anisotropy - its orgins and electrical characteristics, Oilfield Review, 6, 48-56.

Andréis, D. \& MacGregor, L., 2008. Controlled-source electromagnetic sounding in shallow water: principles and applications, Geophysics, 73, F21-F32.

Bhuiyan, A.H., 2009. Three-dimensional modeling and interpretation of CSEM data from offshore Angola, Petrol. Geosci., 15, 175-189.

Bouchara, S. et al., 2015. CSEM anisotropy trends in the Barents Sea, in Proceedings of the 85th SEG Annual Meeting, New Orleans, LA, pp. 879-882.

Brown, V., Hoversten, M., Key, K. \& Chen, J., 2012. Resolution of reservoir scale electrical anisotropy from marine CSEM data, Geophysics, 77, E147-E158.
Chave, A.D., 1984. The Fréchet derivatives of electromagnetic induction, J. geophys. Res., 89, 3373-3380.

Chave, A.D., 2009. On the electromagnetic fields produced by marine frequency domain controlled sources, Geophys. J. Int., 179, 1429-1457.

Chave, A.D., Everett, M.E., Mattsson, J., Boon, J. \& Midgley, J., 2017. On the physics of frequency-domain controlled source electromagnetics in shallow water. 1: isotropic conductivitiy, Geophys. J. Int., 208, 10261042 .

Constable, S.C., 2010. Ten years of marine CSEM for hydrocarbon exploration, Geophysics, 75, 75A67-75A81.

Constable, S.C. \& Srnka, L., 2007. An introduction to marine controlledsource electromagnetic methods for hydrocarbon exploration, Geophysics, 72, WA3-WA12.

Cox, C.S., Kroll, N., Pistek, P. \& Watson, K., 1978. Electromagnetic fields induced by wind waves on the deep-sea floor, J. geophys. Res., 83, 431442.

Eidesmo, T., Ellingsrud, S., MacGregor, L.M., Constable, S., Sinha, M.C., Johanson, S., Kong, F.N. \& Westerdahl, H., 2002. Sea bed logging (SBL), a new method for remote and direct identification of hydrocarbon filled layers in deepwater areas, First Break, 20, 144-152.

Ellingsrud, S., Eidesmo, T., Johansen, S., Sinha, M.C., MacGregor, L.M. \& Constable, S.C., 2002. Remote sensing of hydrocarbon layers by seabed logging (SBL): Results from a cruise offshore, Leading Edge, 21, 872882.

MacGregor, L. \& Tomlinson, J., 2014. Marine controlled-source electromagnetic methods in the hydrocarbon industry: a tutorial on method and practice, Interpretation, 2, SH13-SH32.

Mattsson, J., Engelmark, F. \& Anderson, C., 2013. Towed streamer EM: the challenges of sensitivity and anisotropy, First Break, 31, 155-159.

McKay, A., Mattsson, J. \& Du, Z., 2015. Towed streamer EM - reliable recovery of sub-surface resistivity, First Break, 33, 75-85.

Newman, G.A., Commer, M. \& Carazzone, J.J., 2010. Imaging CSEM data in the presence of electrical anisotropy, Geophysics, 75, F51-F61.

Ramananjaona, C., MacGregor, L. \& Andréis, D., 2011. Sensitivity and inversion of marine electromagnetic data in a vertically anisotropic stratified earth, Geophys. Prosp., 59, 341-360.

Stratton, J.A., 1941. Electromagnetic Theory, p. 615, McGraw-Hill, New York.

Zhdanov, M., Endo, M., Yoon, D., Cuma, M., Mattsson, J. \& Midgley, J., 2014. Anisotropic 3D inversion of towed-streamer electromagnetic data: Case study from the Troll West Oil Province, Interpretation, 2, $1-17$. 